\title{
THE GAUSSIAN CORE MODEL IN HIGH DIMENSIONS
}

\author{
HENRY COHN AND MATTHEW DE COURCY-IRELAND
}

\begin{abstract}
We prove lower bounds for energy in the Gaussian core model, in which point particles interact via a Gaussian potential. Under the potential function $t \mapsto e^{-\alpha t^{2}}$ with $0<\alpha<4 \pi / e$, we show that no point configuration in $\mathbb{R}^{n}$ of density $\rho$ can have energy less than $(\rho+o(1))(\pi / \alpha)^{n / 2}$ as $n \rightarrow \infty$ with $\alpha$ and $\rho$ fixed. This lower bound asymptotically matches the upper bound of $\rho(\pi / \alpha)^{n / 2}$ obtained as the expectation in the Siegel mean value theorem, and it is attained by random lattices. The proof is based on the linear programming bound, and it uses an interpolation construction analogous to those used for the Beurling-Selberg extremal problem in analytic number theory. In the other direction, we prove that the upper bound of $\rho(\pi / \alpha)^{n / 2}$ is no longer asymptotically sharp when $\alpha>\pi e$. As a consequence of our results, we obtain bounds in $\mathbb{R}^{n}$ for the minimal energy under inverse power laws $t \mapsto 1 / t^{n+s}$ with $s>0$, and these bounds are sharp to within a constant factor as $n \rightarrow \infty$ with $s$ fixed.
\end{abstract}

\section{INTRODUCTION}

In the Gaussian core model [34], point particles interact via a Gaussian potential function $t \mapsto e^{-\alpha t^{2}}$, and we wish to minimize the resulting potential energy between them. Specifically, let $\mathcal{C}$ be a discrete subset of $\mathbb{R}^{n}$ with density $\rho>0$. In other words, the number of points of $\mathcal{C}$ in the closed ball $B_{r}^{n}(0)$ of radius $r$ about the origin is asymptotic to $\rho \operatorname{vol}\left(B_{r}^{n}(0)\right)$ as $r \rightarrow \infty$. To define the energy of $\mathcal{C}$ with respect to the potential function $f$, we sum over pairs of points in a large ball:

Definition 1.1. Let $f:(0, \infty) \rightarrow \mathbb{R}$ be any function. The lower $f$-energy of a discrete subset $\mathcal{C}$ of $\mathbb{R}^{n}$ is

$$
\liminf _{r \rightarrow \infty} \frac{1}{\#\left(\mathcal{C} \cap B_{r}^{n}(0)\right)} \sum_{\substack{x, y \in \mathcal{C} \cap B_{r}^{n}(0) \\ x \neq y}} f(|x-y|) .
$$

If this limit exists, and not just the limit inferior, then we call it the $f$-energy of $\mathcal{C}$.

The energy exists whenever $\mathcal{C}$ is periodic and $f$ is sufficiently rapidly decreasing. For example, if $\mathcal{C}$ is a lattice $\Lambda$ in $\mathbb{R}^{n}$ (i.e., a discrete subgroup of rank $n$ ), then its energy is

$$
\sum_{x \in \Lambda \backslash\{0\}} f(|x|) .
$$

In other words, it is a weighted sum over the distances that occur between points in $\Lambda$. In addition to being of interest in physics and geometry [11], this quantity

Date: February 21, 2018.

Matthew de Courcy-Ireland was supported by an internship at Microsoft Research New England and by an NSERC PGS D grant. 
is number-theoretically meaningful [28]. Recall that the theta series of $\Lambda$ is the modular form

$$
\Theta_{\Lambda}(z)=\sum_{x \in \Lambda} e^{\pi i|x|^{2} z}
$$

then the $f$-energy of $\Lambda$ for $f(t)=e^{-\alpha t^{2}}$ is simply $\Theta_{\Lambda}(\alpha i / \pi)-1$.

Given the dimension $n$, density $\rho$, and potential function $f$, how should the points of $\mathcal{C}$ be arranged so as to minimize the energy? We define the minimal energy to be the infimum of the lower energies of all such configurations. See $[10,11]$ for background on ground state and energy minimization problems.

Of course one could consider many sorts of potential functions, but Gaussians play a special role in energy minimization, because they span the cone of completely monotonic functions of squared Euclidean distance (see Theorem 12b in [42, p. 161]). This cone contains all inverse power laws, because

$$
\frac{1}{t^{s}}=\int_{0}^{\infty} e^{-\alpha t^{2}} \frac{\alpha^{s / 2-1}}{\Gamma(s / 2)} d \alpha
$$

for $t>0$ and $s>0$. Thus, lower bounds for Gaussian energy automatically yield corresponding lower bounds for inverse power laws. In number-theoretic terms, (1.1) amounts to the fact that Epstein zeta functions are Mellin transforms of theta functions. See [13] for further justification of why this cone is a natural generalization of inverse power laws.

One can prove the existence of low-energy configurations using the Siegel mean value theorem [32] as follows. There is a canonical probability measure on lattices of determinant 1 (equivalently, density 1 ) in $\mathbb{R}^{n}$, which is characterized by $\mathrm{SL}_{n}(\mathbb{R})$ invariance. For $n>1$, the Siegel mean value theorem says that the expected $f$-energy of a random lattice chosen according to this probability measure is

$$
\int_{\mathbb{R}^{n}} f(|x|) d x
$$

When $f(t)=e^{-\alpha t^{2}}$, the expected energy equals $(\pi / \alpha)^{n / 2}$, and thus there exists a lattice of determinant 1 with energy at most $(\pi / \alpha)^{n / 2}$. The most striking case is when $\alpha=\pi$, in which case the bound becomes 1 . More generally, rescaling shows that there is a lattice of density $\rho$ with energy at most

$$
\rho \int_{\mathbb{R}^{n}} f(|x|) d x=\rho(\pi / \alpha)^{n / 2} .
$$

We call this bound the expectation bound.

It is natural to ask how sharp the expectation bound is. For example, are there configurations in high dimensions with density 1 and energy 0.99 when $\alpha=\pi$ ? We show in this paper that no such configurations exist.

More generally, we prove a matching lower bound for energy in high dimensions under sufficiently wide Gaussian potential functions. Specifically, we will prove asymptotically sharp bounds for energy as $n \rightarrow \infty$ with $\alpha$ and $\rho$ fixed, as long as $\alpha$ is sufficiently small. Our bound is sharp for $\alpha<4 \pi / e=4.62290939 \ldots$ :

Theorem 1.2. When $f(t)=e^{-\alpha t^{2}}$ with $0<\alpha<4 \pi / e$, the minimal $f$-energy in $\mathbb{R}^{n}$ for configurations of density $\rho$ is $(\rho+o(1))(\pi / \alpha)^{n / 2}$ as $n \rightarrow \infty$ with $\alpha$ and $\rho$ fixed, or more generally with $(\alpha, \rho)$ confined to a compact subset of $(0,4 \pi / e) \times(0, \infty)$. 
Note that the $\rho+o(1)$ factor is outside the power of $n / 2$, which makes it a much stronger bound than $\rho(\pi / \alpha+o(1))^{n / 2}$ would be. In particular, when $\alpha=\pi$ the minimal energy converges to $\rho$ as $n \rightarrow \infty$.

For $\alpha<4 \pi / e$, this theorem implies that random lattices are asymptotically optimal for energy. Specifically, a $1-o(1)$ fraction of all lattices must have energy $(\rho+o(1))(\pi / \alpha)^{n / 2}$, because no configuration has energy less than $(\rho+o(1))(\pi / \alpha)^{n / 2}$ and the average over all lattices is $\rho(\pi / \alpha)^{n / 2}$. Thus, imposing the crystalline structure of a lattice does not asymptotically raise the minimal energy compared with amorphous particle arrangements, and no lattice is substantially better than a typical lattice. We do not know whether the same is true when $\alpha \geq 4 \pi / e$.

For fixed $n$ and $\rho$, the Gaussian core model degenerates to the sphere packing problem as $\alpha \rightarrow \infty$ : the energy of a periodic configuration is asymptotically determined by the minimal distance between distinct points, and minimizing energy amounts to maximizing the minimal distance. See Section 9 for a quantitative account of this relationship. The question of whether lattices are near-optimal sphere packings in high dimensions is a major unsolved problem. Our theorem can be viewed as a partial answer, which says that near-optimality of lattices holds for $\alpha<4 \pi / e$. We see no reason to expect it to hold for fixed $n$ as $\alpha \rightarrow \infty$, i.e., in the case of sphere packing, but perhaps it holds for each fixed $\alpha$ as $n \rightarrow \infty$.

When $\alpha \geq 4 \pi / e$, Theorem 1.2 no longer applies, and the best lower bound we know how to prove is the following.

Theorem 1.3. When $f(t)=e^{-\alpha t^{2}}$ with $\alpha \geq 4 \pi / e$, the minimal $f$-energy in $\mathbb{R}^{n}$ for configurations of density $\rho$ is at least

$$
\rho\left(\frac{1}{2} e^{1-\alpha e /(8 \pi)}+o(1)\right)^{n}
$$

as $n \rightarrow \infty$ with $\alpha$ and $\rho$ fixed, or more generally with $(\alpha, \rho)$ confined to a compact subset of $[4 \pi / e, \infty) \times(0, \infty)$.

Note that

$$
\frac{1}{2} e^{1-\alpha e /(8 \pi)}=\sqrt{\frac{\pi}{\alpha}}
$$

when $\alpha=4 \pi / e$, which means the exponential rates in Theorems 1.2 and 1.3 vary continuously as a function of $\alpha$. We deduce those theorems from the following explicit bound:

Theorem 1.4. Let $f: \mathbb{R} \rightarrow \mathbb{R}$ be a Gaussian, let $\lambda_{1}<\lambda_{2}<\cdots$ be the positive roots of the Bessel function $J_{n / 2}$, and choose $r$ so that $\operatorname{vol}\left(B_{r / 2}^{n}(0)\right)=\rho$. Then the minimal $f$-energy for point configurations of density $\rho$ in $\mathbb{R}^{n}$ is at least

$$
\frac{n}{2^{n-1}(n / 2) !^{2}} \sum_{m=1}^{\infty} \frac{\lambda_{m}^{n-2}}{J_{n / 2-1}\left(\lambda_{m}\right)^{2}} f\left(\frac{\lambda_{m}}{\pi r}\right) .
$$

Here $(n / 2)$ ! means $\Gamma(n / 2+1)$ when $n$ is odd, and

$$
\operatorname{vol}\left(B_{r / 2}^{n}(0)\right)=\frac{\pi^{n / 2} r^{n}}{(n / 2) ! 2^{n}} .
$$

Our proof of Theorem 1.4 is based on the linear programming bound from Section 9 of [13], which we prove in slightly greater generality in Proposition 2.2. This lower bound depends on the choice of an auxiliary function, and the best choice 
is not known in general. We obtain Theorem 1.4 by making a specific choice, which is surely suboptimal in general yet still performs well. Our auxiliary function is based on interpolation at Bessel function roots, and it yields the analogue for energy minimization of Proposition 6.1 from [12].

Theorem 1.4 draws no distinction between the cases where $\alpha<4 \pi / e$ and those where $\alpha \geq 4 \pi / e$. Instead, the phase transition between these cases arises in the asymptotic analysis of the bound. We do not know whether the phase transition at $\alpha=4 \pi / e$ reflects an actual change in the behavior of energy minimization at that point, but we can prove that the expectation bound is not sharp when $\alpha$ is large. Specifically, in Proposition 8.2 we obtain an energy upper bound of

$$
\rho\left(\frac{\pi}{\alpha}\right)^{n / 2}\left(e^{1 / 2-\alpha /(2 \pi e)}+o(1)\right)^{n},
$$

which is an exponential improvement on the expectation bound when $\alpha>\pi e$. Thus, Theorem 1.2 covers most of the range of values of $\alpha$ for which the expectation bound could be sharp. We do not know what happens between $4 \pi / e$ and $\pi e$, or whether further improvements are possible when $\alpha>\pi e$.

Because of the uniformity in Theorems 1.2 and 1.3, these theorems can be applied to analyze other potential functions, as long as they are completely monotonic functions of squared distance. In particular, we can use (1.1) to analyze inverse power laws. If $f(t)=1 / t^{n+s}$ with $s>0$, then the expectation bound is infinite but Lemma 8.1 (with $r$ chosen so that $\operatorname{vol}\left(B_{r}^{n}(0)\right)=1 / n$ ) implies that for $\rho$ and $s$ fixed, there exist configurations of density $\rho$ and $f$-energy at most

$$
\left(\frac{2 \rho}{s}+o(1)\right) \frac{\pi^{(n+s) / 2} e^{s / 2}}{\Gamma((n+s) / 2)}
$$

as $n \rightarrow \infty$. Combining Theorem 1.2 with the inequality

$$
\frac{1}{t^{n+s}} \geq \int_{0}^{4 \pi / e} e^{-\alpha t^{2}} \frac{\alpha^{(n+s) / 2-1}}{\Gamma((n+s) / 2)} d \alpha,
$$

which follows from (1.1), proves a lower bound equal to $(2 / e)^{s}+o(1)$ times the upper bound (1.2), which is therefore sharp to within a constant factor as $n \rightarrow \infty$. If the range of validity of Theorem 1.2 could be extended from $4 \pi / e$ to $\pi e$, then the resulting lower bound for inverse power laws would be asymptotically sharp (i.e., the constant factors would match).

Table 1.1 compares our bound from Theorem 1.4 with other bounds when $\alpha=\pi$ and $\rho=1$. The first column shows our bound, the second shows the result of numerically optimizing the auxiliary function in the linear programming bound, and the third shows the lowest energy currently known. The table illustrates the convergence of our bound to $\rho$ as $n \rightarrow \infty$ when $\alpha=\pi$, and it provides evidence that in this case our bound is not much worse than the full linear programming bound.

Aside from the high-dimensional behavior, the most striking aspect of the table is the seemingly matching lower and upper bounds when $n=1,2,8$, or 24 . For $n=1$, Proposition 9.6 from [13] proves a sharp bound, as does Theorem 1.4 in the present paper. When $n=2,8$, or 24 , the agreement between the bounds is a special case of Conjecture 9.4 from [13], which is highly plausible given recent results on sphere packing $[40,14]$. 
TABLE 1.1. A comparison of our lower bound from Theorem 1.4 for energy in $\mathbb{R}^{n}$ when $\alpha=\pi$ and $\rho=1$, the numerically optimized linear programming bound, and the lowest energy currently known (from [15] or the expectation bound).

\begin{tabular}{rlll}
\hline$n$ & Our bound & LP bound & Current record \\
\hline 1 & $0.08643481 \ldots$ & $0.08643481 \ldots$ & $0.08643481 \ldots$ \\
2 & $0.15702654 \ldots$ & $0.15959526 \ldots$ & $0.15959526 \ldots$ \\
3 & $0.21736068 \ldots$ & $0.22321782 \ldots$ & $0.23153532 \ldots$ \\
4 & $0.27028747 \ldots$ & $0.27956960 \ldots$ & $0.28576449 \ldots$ \\
5 & $0.31750042 \ldots$ & $0.33011740 \ldots$ & $0.34868410 \ldots$ \\
6 & $0.36010894 \ldots$ & $0.37587226 \ldots$ & $0.38874675 \ldots$ \\
7 & $0.39889096 \ldots$ & $0.41756856 \ldots$ & $0.42445404 \ldots$ \\
8 & $0.43442005 \ldots$ & $0.45576289 \ldots$ & $0.45576289 \ldots$ \\
9 & $0.46713560 \ldots$ & $0.49089167 \ldots$ & $0.49771252 \ldots$ \\
24 & $0.76270306 \ldots$ & $0.79965280 \ldots$ & $0.79965280 \ldots$ \\
100 & $0.99321117 \ldots$ & $0.99735690 \ldots$ & 1 \\
200 & $0.99991895 \ldots$ & $0.99998973 \ldots$ & 1 \\
500 & $0.99999999 \ldots$ & $0.99999999 \ldots$ & 1 \\
\hline
\end{tabular}

Theorem 1.4 is analogous to the results of [3] for spherical codes, and as in that paper we prove a corresponding optimality result under certain conditions (Proposition 6.3). Our construction of the auxiliary function for the linear programming bound is also analogous to the solution of the Beurling-Selberg extremal problem in analytic number theory [5, 6, 7, 8, 19, 20, 37], which arose independently in Selberg's work on sieve theory and Beurling's work on analytic functions (see [31, p. 226] for historical comments). In particular, the relevant case for our work is Gaussian subordination $[6,5]$. In that problem, we are given parameters $n, \alpha$, and $r$, and the goal is to find the entire function $h$ of exponential type at most $2 \pi r$ such that $h$ maps $\mathbb{R}$ to $\mathbb{R}, h(t) \leq e^{-\alpha t^{2}}$ for all $t \in \mathbb{R}$, and

$$
\int_{\mathbb{R}^{n}} h(|x|) d x
$$

is maximized. This goal is achieved in $[6,5]$. Optimizing the auxiliary function in the linear programming bound involves somewhat different constraints, but we construct our auxiliary function using a similar approach based on interpolation, and our proof techniques can be used to give a new proof of Theorems 2 and 3 from [6] and their higher-dimensional analogues from [5]. Our results are also similar to Poltyrev's work [26] on communicating over a channel with Gaussian random noise, in which he determined the exact error exponents for a certain parameter range and showed that they are achieved by random lattices.

In the remainder of this paper, we first develop the linear programming bound in Section 2. In Section 3 we prove a key lemma about positive-definite functions, and in Section 4 we recall some background about entire functions of exponential type and formulate a strategy for proving Theorem 1.4. We then apply these results to prove Theorem 1.4 via Hermite interpolation in Sections 5 and 6. In Section 7, we carry out an asymptotic analysis of this bound to deduce Theorems 1.2 and 1.3. We derive an improved upper bound for energy in Section 8. Finally, we draw a 
more concrete connection between sphere packing and the Gaussian core model in Section 9, and we conclude with open problems in Section 10.

\section{ACKNOWLEDGMEnts}

We thank Ganesh Ajjanagadde, Peter Sarnak, and the anonymous referees for their helpful comments on the manuscript.

\section{THE LINEAR PROGRAMMing BOUND}

In this section we develop the linear programming bound for energy minimization. This bound is essentially Proposition 9.3 of [13], but the proof given there works only for periodic configurations. Here we extend it to arbitrary configurations.

Definition 2.1. A continuous function $h: \mathbb{R}^{n} \rightarrow \mathbb{C}$ is positive definite if $h(-x)=$ $\overline{h(x)}$ for all $x \in \mathbb{R}^{n}$, and for all $N \in \mathbb{N}$ and $x_{1}, \ldots, x_{N} \in \mathbb{R}^{n}$, the $N \times N$ Hermitian matrix

is positive semidefinite.

$$
\left(h\left(x_{j}-x_{k}\right)\right)_{1 \leq j, k \leq N}
$$

The latter condition is equivalent to asserting that for all $x_{1}, \ldots, x_{N} \in \mathbb{R}^{n}$ and $t_{1}, \ldots, t_{N} \in \mathbb{C}$

$$
\sum_{1 \leq j, k \leq N} t_{j} \overline{t_{k}} h\left(x_{j}-x_{k}\right) \geq 0
$$

We will need several properties of positive-definite functions. They are closed under multiplication, by the Schur product theorem (Theorem 7.5.3 in [22]), which says that positive-semidefinite matrices are closed under the Hadamard product. Furthermore, if $h$ is positive definite, then the $2 \times 2$ matrix

$$
\left[\begin{array}{cc}
h(0) & h(x) \\
h(-x) & h(0)
\end{array}\right]
$$

is positive semidefinite for every $x \in \mathbb{R}^{n}$, from which it follows that $h(0) \geq 0$ and $|h(x)| \leq h(0)$ by taking the determinant.

Recall that Bochner's theorem characterizes positive-definite functions as those of the form

$$
x \mapsto \int_{\mathbb{R}^{n}} e^{2 \pi i\langle x, y\rangle} d \mu(y),
$$

where $\mu$ is a finite measure on $\mathbb{R}^{n}$ with respect to the Borel $\sigma$-algebra (see Theorem 6.6.6 in [33]). Define the Fourier transform $\widehat{h}$ of an integrable function $h: \mathbb{R}^{n} \rightarrow \mathbb{C}$ by

$$
\widehat{h}(y)=\int_{\mathbb{R}^{n}} h(x) e^{-2 \pi i\langle x, y\rangle} d x .
$$

Then it follows from Bochner's theorem and Fourier inversion that if $h$ and $\widehat{h}$ are both integrable, then $h$ is positive definite if and only if $\widehat{h} \geq 0$.

The linear programming bound can be stated as follows. As mentioned above, it is essentially Proposition 9.3 from [13], but we state it here in slightly greater generality and give a different proof. 
Proposition 2.2 (Cohn and Kumar [13]). Let $f:(0, \infty) \rightarrow \mathbb{R}$ be any function, and suppose $h: \mathbb{R}^{n} \rightarrow \mathbb{R}$ is continuous, positive definite, and integrable. If $h(x) \leq f(|x|)$ for all $x \in \mathbb{R}^{n} \backslash\{0\}$, then every subset of $\mathbb{R}^{n}$ with density $\rho$ has lower $f$-energy at least $\rho \widehat{h}(0)-h(0)$.

The proof will follow the approach used to prove Theorem 3.3 in [17].

Proof. Let $\mathcal{C}$ be a subset of $\mathbb{R}^{n}$ of density $\rho$. For each $r>0$, let

$$
\mathcal{C}_{r}=\{x \in \mathcal{C}:|x| \leq r\},
$$

let $N_{r}=\# \mathcal{C}_{r}$, and let $V_{r}=\operatorname{vol}\left(B_{r}^{n}(0)\right)$. Then

$$
\lim _{r \rightarrow \infty} \frac{N_{r}}{V_{r}}=\rho
$$

To avoid dividing by zero when computing energy, we will restrict our attention to $r$ such that $N_{r}>0$.

The proof will be based on renormalizing a sum over $\mathcal{C}$ by subtracting a uniform background density. Specifically, we will consider the signed measure

$$
\nu=\sum_{x \in \mathcal{C}_{r}} \delta_{x}-\frac{N_{r}}{V_{r}} \mu_{R}
$$

where $\delta_{x}$ denotes a delta function at $x, \mu_{R}$ denotes Lebesgue measure on the ball of radius $R$ centered at the origin, and $R=r+\sqrt{r}$. The shift by $\sqrt{r}$ simplifies one of the limits we need below, but it is not conceptually important. Ignoring this shift, observe that

$$
\sum_{x \in \mathcal{C}_{r}} \delta_{x}-\frac{N_{r}}{V_{r}} \mu_{r}
$$

has integral zero, which explains the factor of $N_{r} / V_{r}$.

Because $h$ is positive definite, it follows from approximating integrals with respect to $\mu_{R}$ by Riemann sums and applying (2.1) that

$$
\iint h(x-y) d \nu(x) d \nu(y) \geq 0 .
$$

Equivalently,

$$
\frac{N_{r}^{2}}{V_{r}^{2}} \iint_{|x|,|y| \leq R} h(x-y) d x d y-\frac{2 N_{r}}{V_{r}} \sum_{x \in \mathcal{C}_{r}} \int_{|y| \leq R} h(x-y) d y+\sum_{x, y \in \mathcal{C}_{r}} h(x-y) \geq 0 .
$$

Applying the inequality $h(x-y) \leq f(|x-y|)$ and rearranging yields

$$
\begin{aligned}
\frac{1}{N_{r}} \sum_{\substack{x, y \in \mathcal{C}_{r} \\
x \neq y}} f(|x-y|) \geq & \frac{2 N_{r}}{V_{r}} \cdot \frac{1}{N_{r}} \sum_{x \in \mathcal{C}_{r}} \int_{|y| \leq R} h(x-y) d y-h(0) \\
& -\frac{N_{r}}{V_{r}} \cdot \frac{1}{V_{r}} \iint_{|x|,|y| \leq R} h(x-y) d x d y .
\end{aligned}
$$

To complete the proof, we will show that the right side of (2.2) converges to $\rho \widehat{h}(0)-h(0)$ as $r \rightarrow \infty$. First, we claim that

$$
\frac{1}{N_{r}} \sum_{x \in \mathcal{C}_{r}} \int_{|y| \leq R} h(x-y) d y \rightarrow \widehat{h}(0),
$$


for the following reason. Each fixed summand converges to $\widehat{h}(0)$, because

$$
\int_{|y| \leq R} h(x-y) d y \rightarrow \int_{\mathbb{R}^{n}} h(x-y) d y=\widehat{h}(0),
$$

and all we need to verify is that this limit holds uniformly for $x \in \mathcal{C}_{r}$. To check the uniformity, we note that $\{x-y:|y| \leq R\}$ contains $B_{R-|x|}^{n}(0)$. In particular, because $|x| \leq r$ and $R-r=\sqrt{r} \rightarrow \infty$, the integrals

$$
\int_{|y| \leq R} h(x-y) d y
$$

include all values of $x-y$ of length at most $\sqrt{r}$. Thus, they converge uniformly to $\widehat{h}(0)$. Similarly,

$$
\frac{1}{V_{r}} \iint_{|x|,|y| \leq R} h(x-y) d x d y \rightarrow \widehat{h}(0),
$$

since for $|x| \leq r$ the $y$-integrals converge uniformly to $\widehat{h}(0)$, and the contributions from $r \leq|x| \leq r+\sqrt{r}$ are negligible compared with $V_{r}$. Combining these limits with $N_{r} / V_{r} \rightarrow \rho$ and (2.2) yields

$$
\liminf _{r \rightarrow \infty} \frac{1}{N_{r}} \sum_{\substack{x, y \in \mathcal{C}_{r} \\ x \neq y}} f(|x-y|) \geq \rho \widehat{h}(0)-h(0),
$$

as desired.

\section{Positive-definite functions}

The proof of Theorem 1.4 will make crucial use of the following proposition, which says that removing successive roots from a suitable Bessel function always yields a positive-definite function.

Proposition 3.1. Let $\nu=n / 2-1$, and let $\lambda_{1}<\lambda_{2}<\cdots$ be the positive roots of $J_{\nu}$. Then for each $k \geq 0$, the function

$$
x \mapsto \frac{J_{\nu}(|x|)}{|x|^{\nu}\left(1-\frac{|x|^{2}}{\lambda_{1}^{2}}\right) \ldots\left(1-\frac{|x|^{2}}{\lambda_{k}^{2}}\right)}
$$

is positive definite on $\mathbb{R}^{n}$ (where of course we define the function by continuity when its denominator vanishes).

This assertion is well known for $k=0$, and it is not hard to prove it for $k=1$ using the Christoffel-Darboux formula. For general $k$, it is a Bessel function analogue of Theorem 3.1 from [13]:

Theorem 3.2 (Cohn and Kumar [13]). Let $p_{0}, \ldots, p_{n}$ be the monic orthogonal polynomials with respect to some measure on $\mathbb{R}$, where $\operatorname{deg} p_{i}=i$, let $\alpha \in \mathbb{R}$, and let

$$
r_{1}<r_{2}<\cdots<r_{n}
$$

be the roots of $p_{n}+\alpha p_{n-1}$. Then for $1 \leq k<n$, the polynomial

$$
\prod_{i=1}^{k}\left(t-r_{k}\right)
$$

has positive coefficients in terms of $p_{0}(t), \ldots, p_{k}(t)$. 
Proposition 3.1 could very likely be proved by adapting the proof given in [13], but we will deduce it from this theorem by taking a suitable limit of polynomials.

The polynomials we will use are those that are positive definite on the unit sphere $S^{n-1}$ in $\mathbb{R}^{n}$. Recall that a function $p:[-1,1] \rightarrow \mathbb{R}$ is positive definite on $S^{n-1}$ if for all $N \in \mathbb{N}$ and $x_{1}, \ldots, x_{N} \in S^{n-1}$, the $N \times N$ matrix

$$
\left(p\left(\left\langle x_{i}, x_{j}\right\rangle\right)\right)_{1 \leq i, j \leq N}
$$

is positive semidefinite. ${ }^{1}$ Schoenberg's theorem [30] characterizes positive-definite functions on $S^{n-1}$ as the nonnegative linear combinations of the Jacobi polynomials $P_{m}^{((n-3) / 2,(n-3) / 2)}$ with $m \geq 0$. Jacobi polynomials with these parameters are also known as Gegenbauer polynomials or ultraspherical polynomials.

We will deduce Proposition 3.1 from Theorem 3.2 applied to the Jacobi polynomials $P_{m}^{(\nu, \nu)}$. Note that the parameter shift from $(n-3) / 2$ to $\nu=n / 2-1$ means that these polynomials are positive definite on $S^{n}$, not just $S^{n-1}$.

Proof of Proposition 3.1. What we must prove is that for all $x_{1}, \ldots, x_{N} \in \mathbb{R}^{n}$, the $N \times N$ matrix whose $i, j$ entry is

$$
\frac{J_{\nu}\left(\left|x_{i}-x_{j}\right|\right)}{\left|x_{i}-x_{j}\right|^{\nu}\left(1-\frac{\left|x_{i}-x_{j}\right|^{2}}{\lambda_{1}^{2}}\right) \ldots\left(1-\frac{\left|x_{i}-x_{j}\right|^{2}}{\lambda_{k}^{2}}\right)}
$$

is positive semidefinite.

To reduce from Bessel functions to Jacobi polynomials, we will use the MehlerHeine formula for Jacobi polynomials (Theorem 8.1.1 in [35]), which says that

$$
\lim _{m \rightarrow \infty}(2 m)^{-\nu} P_{m}^{(\nu, \nu)}\left(1-\frac{z^{2}}{2 m^{2}}\right)=z^{-\nu} J_{\nu}(z),
$$

uniformly for $z$ in any compact subset of $\mathbb{C}$. Furthermore, Hurwitz's theorem implies that if $r_{1, m}>\cdots>r_{m, m}$ are the roots of $P_{m}^{(\nu, \nu)}$, then

$$
r_{i, m}=1-\frac{\lambda_{i}^{2}}{2 m^{2}}+o\left(1 / m^{2}\right)
$$

for each fixed $i$ as $m \rightarrow \infty$ (Theorem 8.1.2 in [35]).

To take advantage of the fact that the polynomials $P_{m}^{(\nu, \nu)}$ are positive definite on the unit sphere $S^{n}$, we will map $\mathbb{R}^{n}$ to $S^{n}$. We view $\mathbb{R}^{n}$ as a hyperplane in $\mathbb{R}^{n+1}$. Let $e_{n}$ be a unit vector in $\mathbb{R}^{n+1}$ orthogonal to $\mathbb{R}^{n}$, and for each positive integer $m$ define a function $f_{m}: \mathbb{R}^{n} \rightarrow S^{n}$ by

$$
f_{m}(x)=\frac{e_{n}+x / m}{\sqrt{1+|x|^{2} / m^{2}}} .
$$

Then a short calculation shows that

$$
\left\langle f_{m}(x), f_{m}(y)\right\rangle=1-\frac{|x-y|^{2}}{2 m^{2}}+O\left(1 / m^{4}\right),
$$

where the big- $O$ term is uniform when $x$ and $y$ are confined to a compact set. It now follows that for all $x, y \in \mathbb{R}^{n}$,

$$
\lim _{m \rightarrow \infty}(2 m)^{-\nu} P_{m}^{(\nu, \nu)}\left(\left\langle f_{m}(x), f_{m}(y)\right\rangle\right)=|x-y|^{-\nu} J_{\nu}(|x-y|) .
$$

\footnotetext{
${ }^{1}$ Strictly speaking, it is the function $\widetilde{p}: S^{n-1} \times S^{n-1} \rightarrow \mathbb{R}$ defined by $\widetilde{p}(x, y)=p(\langle x, y\rangle)$ that is positive definite, but it is more convenient to talk about $p$.
} 
In particular, the $O\left(1 / \mathrm{m}^{4}\right)$ error term is handled by the uniformity of convergence in the Mehler-Heine formula.

By Theorem 3.2, the polynomial

$$
\frac{P_{m}^{(\nu, \nu)}(z)}{\left(z-r_{1, m}\right) \ldots\left(z-r_{k, m}\right)}
$$

is a linear combination of $P_{0}^{(\nu, \nu)}(z), \ldots, P_{m-k}^{(\nu, \nu)}(z)$ with nonnegative coefficients for each $k \leq m$. It follows that this polynomial is positive definite on $S^{n}$. Thus, for all $x_{1}, \ldots, x_{N} \in \mathbb{R}^{n}$, the $N \times N$ matrix with $i, j$ entry

$$
\frac{P_{m}^{(\nu, \nu)}\left(\left\langle f_{m}\left(x_{i}\right), f_{m}\left(x_{j}\right)\right\rangle\right)}{\left(\left\langle f_{m}\left(x_{i}\right), f_{m}\left(x_{j}\right)\right\rangle-r_{1, m}\right) \ldots\left(\left\langle f_{m}\left(x_{i}\right), f_{m}\left(x_{j}\right)\right\rangle-r_{k, m}\right)}
$$

is positive semidefinite.

It follows from (3.2) and (3.3) that for $1 \leq \ell \leq k$,

$$
\lim _{m \rightarrow \infty} \frac{2 m^{2}}{\lambda_{\ell}^{2}}\left(\left\langle f_{m}\left(x_{i}\right), f_{m}\left(x_{j}\right)\right\rangle-r_{\ell, m}\right)=1-\frac{\left|x_{i}-x_{j}\right|^{2}}{\lambda_{\ell}^{2}} .
$$

Combining this limit with the Mehler-Heine formula (3.1), we find that

$$
\lim _{m \rightarrow \infty}\left(\frac{2^{k-\nu} m^{2 k-\nu}}{\lambda_{1}^{2} \ldots \lambda_{k}^{2}} \cdot \frac{P_{m}^{(\nu, \nu)}\left(\left\langle f_{m}\left(x_{i}\right), f_{m}\left(x_{j}\right)\right\rangle\right)}{\left(\left\langle f_{m}\left(x_{i}\right), f_{m}\left(x_{j}\right)\right\rangle-r_{1, m}\right) \ldots\left(\left\langle f_{m}\left(x_{i}\right), f_{m}\left(x_{j}\right)\right\rangle-r_{k, m}\right)}\right)
$$

is equal to

$$
\frac{J_{\nu}\left(\left|x_{i}-x_{j}\right|\right)}{\left|x_{i}-x_{j}\right|^{\nu}\left(1-\frac{\left|x_{i}-x_{j}\right|^{2}}{\lambda_{1}^{2}}\right) \ldots\left(1-\frac{\left|x_{i}-x_{j}\right|^{2}}{\lambda_{k}^{2}}\right)},
$$

as long as $\left|x_{i}-x_{j}\right|$ is not among $\lambda_{1}, \ldots, \lambda_{k}$. Applying this limit to the matrix from the previous paragraph shows that the $N \times N$ matrix with entries (3.4) is positive semidefinite, again assuming $\left|x_{i}-x_{j}\right|$ is never among $\lambda_{1}, \ldots, \lambda_{k}$.

All that remains is to deal with the case in which $\left|x_{i}-x_{j}\right|=\lambda_{\ell}$ for some $i, j$, and $\ell$. However, that case follows easily by continuity: (3.4) is a continuous function of $\left|x_{i}-x_{j}\right|$, and every configuration $x_{1}, \ldots, x_{N}$ has arbitrarily small perturbations in which $\lambda_{1}, \ldots, \lambda_{k}$ do not occur as distances.

\section{EntiRe FunCtions of EXPONENTIAL TYPE}

Recall that one version of the Paley-Wiener theorem characterizes Fourier transforms of compactly supported distributions as entire functions of exponential type:

Proposition 4.1. Let $h$ be a function from $[0, \infty)$ to $\mathbb{R}$. Then the radial function $x \mapsto h(|x|)$ on $\mathbb{R}^{n}$ is the Fourier transform of a distribution supported on $B_{r}^{n}(0)$ if and only if $h$ extends to an even entire function on $\mathbb{C}$ for which there are constants $C$ and $k$ such that

$$
|h(z)| \leq C(1+|z|)^{k} e^{2 \pi r|\operatorname{Im} z|}
$$

for all $z \in \mathbb{C}$.

In particular, $h$ is an entire function of exponential type at most $2 \pi r$. For a proof of Proposition 4.1, see Theorem 7.3.1 in [21], together with Lemma 3.4 in [9] for information on how to obtain this result as a special case of the theorem from [21]. 
Corollary 4.2. Let $\nu=n / 2-1$, and let $\lambda_{1}<\lambda_{2}<\cdots$ be the positive roots of $J_{\nu}$. Then for each $k$, the function

$$
x \mapsto \frac{J_{\nu}(|x|)}{|x|^{\nu}\left(1-\frac{|x|^{2}}{\lambda_{1}^{2}}\right) \ldots\left(1-\frac{|x|^{2}}{\lambda_{k}^{2}}\right)}
$$

on $\mathbb{R}^{n}$ has Fourier transform supported in $B_{1 /(2 \pi)}^{n}(0)$.

Note that these functions are the same as those in Proposition 3.1. When $k$ is small the functions are not integrable, in which case we take their Fourier transforms as tempered distributions.

Proof. For $k=0$, the function is $x \mapsto J_{\nu}(|x|) /|x|^{\nu}$, and up to scaling it is the Fourier transform of a delta function supported on the sphere of radius $1 /(2 \pi)$ about the origin, by the Bessel function formula for the radial Fourier transform (Theorem 9.10.3 in [1]). For $k \geq 1$, the additional factors in the denominator do not disrupt the growth condition from Proposition 4.1.

We will need the following summation formula for entire functions of exponential type:

Lemma 4.3 (Ben Ghanem and Frappier [2]). Let $h$ be an even entire function of exponential type at most $2 \pi r$, and suppose that $h(x)=O\left((1+|x|)^{-n-\delta}\right)$ for $x \in \mathbb{R}$ with $\delta>0$. If $\lambda_{1}<\lambda_{2}<\cdots$ are the positive roots of the Bessel function $J_{n / 2}$, then

$$
\frac{n}{2^{n-1}(n / 2) !^{2}} \sum_{m=1}^{\infty} \frac{\lambda_{m}^{n-2}}{J_{n / 2-1}\left(\lambda_{m}\right)^{2}} h\left(\frac{\lambda_{m}}{\pi r}\right)=\operatorname{vol}\left(B_{r / 2}^{n}(0)\right) \int_{\mathbb{R}^{n}} h(|x|) d x-h(0) .
$$

This lemma is the special case of Theorem 2 in [2] with $p=0$ and $\alpha=n / 2$ (see also Theorem 3.1 in [9]). Note that the decay exponent $n+\delta>n$ is enough to ensure absolute convergence of the infinite sum. Indeed, the denominator satisfies $J_{n / 2-1}\left(\lambda_{m}\right)^{2} \sim 2 /\left(\pi \lambda_{m}\right)$, because $J_{\nu}(x)^{2}+J_{\nu+1}(x)^{2} \sim 2 /(\pi x)$ as $x \rightarrow \infty$ (see $\S 7.21$ of $\left[41\right.$, p. 200]). Since $\lambda_{m}$ grows linearly with $m$, the summands are dominated by $m^{-1-\delta}$, which is summable.

We can now formulate our strategy for the proof of Theorem 1.4. Let $f: \mathbb{R} \rightarrow \mathbb{R}$ be a Gaussian, and choose $\lambda_{1}<\lambda_{2}<\cdots$ and $r$ as in Theorem 1.4. We will construct a radial auxiliary function $h: \mathbb{R}^{n} \rightarrow \mathbb{R}$ such that

(1) the function $h$ is continuous, integrable, and positive definite,

(2) $h(x) \leq f(|x|)$ for all $x \in \mathbb{R}^{n}$,

(3) $h(x)=O\left((1+|x|)^{-(n+1)}\right)$,

(4) the support of $\widehat{h}$ is contained in $B_{r}^{n}(0)$, and

(5) the functions $f$ and $h$ agree at radius $\lambda_{m} /(\pi r)$ for each $m \geq 1$.

We write $h\left(\lambda_{m} /(\pi r)\right)$ to denote the common value of $h$ at radius $\lambda_{m} /(\pi r)$, so that (5) can be rephrased as

$$
h\left(\frac{\lambda_{m}}{\pi r}\right)=f\left(\frac{\lambda_{m}}{\pi r}\right) .
$$

If we use this auxiliary function $h$, then the linear programming bound tells us that the lower $f$-energy for configurations of density $\rho$ in $\mathbb{R}^{n}$ is at least $\rho \widehat{h}(0)-h(0)$. 
Given our choice of $r$, Lemma 4.3 implies that

$$
\begin{aligned}
\rho \widehat{h}(0)-h(0) & =\frac{n}{2^{n-1}(n / 2) !^{2}} \sum_{m=1}^{\infty} \frac{\lambda_{m}^{n-2}}{J_{n / 2-1}\left(\lambda_{m}\right)^{2}} h\left(\frac{\lambda_{m}}{\pi r}\right) \\
& =\frac{n}{2^{n-1}(n / 2) !^{2}} \sum_{m=1}^{\infty} \frac{\lambda_{m}^{n-2}}{J_{n / 2-1}\left(\lambda_{m}\right)^{2}} f\left(\frac{\lambda_{m}}{\pi r}\right),
\end{aligned}
$$

as desired. All that remains is to construct $h$.

\section{INTERPOLATION BY POLYNOMIALS}

To complete the proof of Theorem 1.4, we must construct an auxiliary function $h$ with properties (1) through (5) from the previous section. In this section, we lay the groundwork by studying polynomial interpolation.

Recall that in Hermite interpolation, we are given distinct points $t_{1}, \ldots, t_{N} \in \mathbb{R}$ and multiplicities $k_{1}, \ldots, k_{N} \in \mathbb{N}$, and we wish to construct the unique polynomial $p$ of degree less than $k_{1}+\cdots+k_{N}$ with given values $p^{(k)}\left(t_{j}\right)$ for $1 \leq j \leq N$ and $0 \leq k<k_{j}$. In other words, we specify $p$ to order $k_{j}$ at each point $t_{j}$. By the Hermite interpolation of a function $f$ we mean that $f$ is used to specify the values of $p$, i.e., $p^{(k)}\left(t_{j}\right)=f^{(k)}\left(t_{j}\right)$. See Section 2.1 of [13] for a brief review of Hermite interpolation. We will typically indicate the multiplicities $k_{1}, \ldots, k_{N}$ by specifying a multiset of interpolation points

$$
\overbrace{t_{1}, \ldots, t_{1}}^{k_{1}}, \overbrace{t_{2}, \ldots, t_{2}}^{k_{2}}, \ldots, \overbrace{t_{N}, \ldots, t_{N}}^{k_{N}},
$$

with multiplicities indicated by repetition.

We will obtain our auxiliary function $h$ as a limit of Hermite interpolation polynomials. It will prove convenient to apply a quadratic change of variables $z=-t^{2}$, so that the Gaussian $e^{-\alpha t^{2}}$ becomes the exponential function $e^{\alpha z}$.

Fix $\alpha>0$, and define $\lambda_{1}<\lambda_{2}<\cdots$ and $r$ as in Theorem 1.4. We define the sequence of interpolation points $u_{1}, u_{2}, \ldots$ by

$$
u_{2 j-1}=u_{2 j}=-\left(\frac{\lambda_{j}}{\pi r}\right)^{2}
$$

for $j \geq 1$. Aside from the quadratic change of variables, these are exactly the points at which we wish the auxiliary function to equal the Gaussian, and the repetition indicates that we will interpolate to second order. Note that $\left|u_{j}\right| \asymp j^{2}$ as $j \rightarrow \infty$, in the sense that there are positive constants $c$ and $C$ (depending on $n$ but not $j$ ) such that $c j^{2}<\left|u_{j}\right|<C j^{2}$ for all $j$.

Let $p_{M}$ be the Hermite interpolation of the function $z \mapsto e^{\alpha z}$ at $u_{1}, \ldots, u_{M}$. We will define the auxiliary function $h$ by

$$
h(w)=\lim _{M \rightarrow \infty} p_{M}\left(-|w|^{2}\right)
$$

for $w \in \mathbb{R}^{n}$, once we show that this limit exists.

The following simple algebraic lemma will play an important role in the proof. In the lemma, we introduce a new variable $u_{0}$. It will play a similar role to that of $u_{1}, u_{2}, \ldots$ (as the notation suggests), but we can choose its value arbitrarily. 
Lemma 5.1. If $u_{0}$ and $z$ are complex numbers such that $z \notin\left\{u_{0}, \ldots, u_{M}\right\}$, then

$$
\frac{1}{z-u_{0}}=\sum_{k=0}^{M} \frac{1}{z-u_{k}} \prod_{j=k+1}^{M} \frac{1-u_{0} / u_{j}}{1-z / u_{j}} .
$$

If $z \notin\left\{u_{0}, u_{1}, \ldots\right\}$, then

$$
\frac{1}{z-u_{0}}=\sum_{k=0}^{\infty} \frac{1}{z-u_{k}} \prod_{j=k+1}^{\infty} \frac{1-u_{0} / u_{j}}{1-z / u_{j}} .
$$

The only dependence of this lemma on our choice of $u_{1}, u_{2}, \ldots$ is through their quadratic growth, which will justify taking the limit as $M \rightarrow \infty$.

Proof. The first identity is trivial for $M=0$, and we will prove it by induction on $M$. After clearing denominators, it amounts to

$$
\prod_{j=1}^{M}\left(z-u_{j}\right)=\sum_{k=0}^{M} \prod_{j=0}^{k-1}\left(z-u_{j}\right) \prod_{j=k+1}^{M}\left(u_{0}-u_{j}\right) .
$$

If we separate out the terms with $k=M$ and $j=M$ and apply the induction hypothesis, we find that the right side is

$$
\left(z-u_{1}\right) \ldots\left(z-u_{M-1}\right)\left(u_{0}-u_{M}\right)+\left(z-u_{0}\right)\left(z-u_{1}\right) \ldots\left(z-u_{M-1}\right),
$$

which simplifies as desired.

To prove the second identity, we take the limit as $M \rightarrow \infty$. Because $\left|u_{j}\right|$ grows quadratically as a function of $j$, the sum

$$
\sum_{k=1}^{\infty} \frac{1}{\left|z-u_{k}\right|}
$$

is finite, and the products

$$
\prod_{j=k+1}^{\infty} \frac{1-u_{0} / u_{j}}{1-z / u_{j}}
$$

converge and are thus uniformly bounded as a function of $k$. It follows that

$$
\sum_{k=0}^{M} \frac{1}{z-u_{k}} \prod_{j=k+1}^{M} \frac{1-u_{0} / u_{j}}{1-z / u_{j}}-\sum_{k=0}^{M} \frac{1}{z-u_{k}} \prod_{j=k+1}^{\infty} \frac{1-u_{0} / u_{j}}{1-z / u_{j}}
$$

equals

$$
\sum_{k=0}^{M} \frac{1}{z-u_{k}}\left(\prod_{j=k+1}^{M} \frac{1-u_{0} / u_{j}}{1-z / u_{j}}\right)\left(1-\prod_{j=M+1}^{\infty} \frac{1-u_{0} / u_{j}}{1-z / u_{j}}\right)
$$




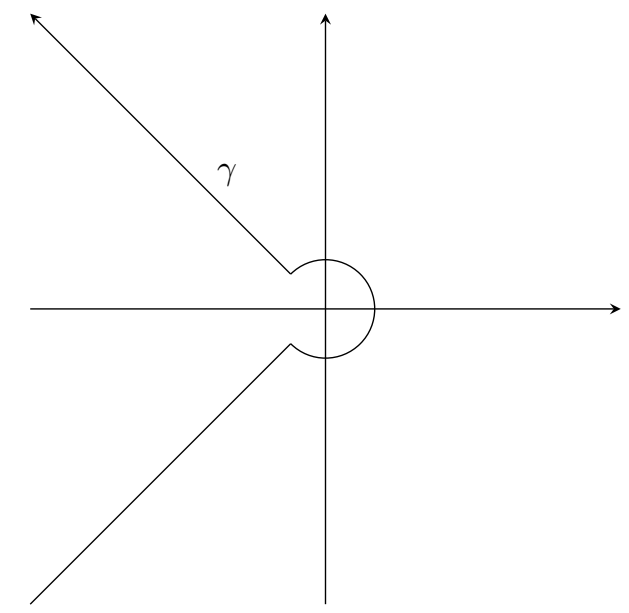

FiguRE 5.1. The contour $\gamma$, oriented counterclockwise, together with the coordinate axes. The circular arc is part of the unit circle, and the rays are at $45^{\circ}$ angles from the axes.

which converges to 0 as $M \rightarrow \infty$ because the first factor in parentheses remains bounded while the second converges to 0 . Thus,

$$
\begin{aligned}
\frac{1}{z-u_{0}} & =\lim _{M \rightarrow \infty} \sum_{k=0}^{M} \frac{1}{z-u_{k}} \prod_{j=k+1}^{M} \frac{1-u_{0} / u_{j}}{1-z / u_{j}} \\
& =\lim _{M \rightarrow \infty} \sum_{k=0}^{M} \frac{1}{z-u_{k}} \prod_{j=k+1}^{\infty} \frac{1-u_{0} / u_{j}}{1-z / u_{j}} \\
& =\sum_{k=0}^{\infty} \frac{1}{z-u_{k}} \prod_{j=k+1}^{\infty} \frac{1-u_{0} / u_{j}}{1-z / u_{j}}
\end{aligned}
$$

as desired.

We will obtain the interpolating polynomial $p_{M}$ from Lemma 5.1, thereby recovering a standard contour integral representation for Hermite interpolation. In the following calculations, we will use $u_{0}$ as the variable for our interpolating polynomial, while $u_{1}, u_{2}, \ldots$ will be fixed interpolation nodes.

Let $\gamma$ be the contour in the complex plane that traces the points $x+i x$ for $x$ from $-\infty$ to $-\sqrt{2} / 2$, wraps counterclockwise around the unit circle to $-\sqrt{2} / 2+i \sqrt{2} / 2$, and then traces the points $-x+i x$ for $x$ from $\sqrt{2} / 2$ to $\infty$, as shown in Figure 5.1.

Integrating

$$
\frac{1}{2 \pi i} e^{\alpha z} \frac{d z}{z-u_{0}}
$$

around $\gamma$ and applying the first identity from Lemma 5.1 shows that for $u_{0} \in(-\infty, 0]$,

$$
e^{\alpha u_{0}}=\sum_{k=0}^{M} H_{k, M} \prod_{j=k+1}^{M}\left(1-\frac{u_{0}}{u_{j}}\right),
$$


where

$$
H_{k, M}=\frac{1}{2 \pi i} \int_{\gamma} \frac{e^{\alpha z} d z}{\left(z-u_{k}\right) \prod_{j=k+1}^{M}\left(1-z / u_{j}\right)} .
$$

Note that the reason we use an unbounded contour $\gamma$ is to avoid having it depend on $M$; the use of $\gamma$ is justified by the exponential decay of $e^{\alpha z}$ as $\operatorname{Re} z \rightarrow-\infty$.

For $k \geq 1$, the coefficient $H_{k, M}$ is a constant independent of $u_{0}$, while $H_{0, M}$ is a holomorphic function of $u_{0}$. It follows from (5.2) that

$$
p_{M}\left(u_{0}\right)=\sum_{k=1}^{M} H_{k, M} \prod_{j=k+1}^{M}\left(1-\frac{u_{0}}{u_{j}}\right),
$$

because

$$
e^{\alpha u_{0}}-\sum_{k=1}^{M} H_{k, M} \prod_{j=k+1}^{M}\left(1-\frac{u_{0}}{u_{j}}\right)=H_{0, M} \prod_{j=1}^{M}\left(1-\frac{u_{0}}{u_{j}}\right),
$$

which vanishes to the desired order (i.e., the multiplicity) at each of $u_{1}, \ldots, u_{M}$, and $p_{M}$ is the unique polynomial of degree less than $M$ with this property.

Lemma 5.2 (Cohn and Woo [16]). Let $t_{1}, \ldots, t_{M}$ be elements of the interval $I$, not necessarily distinct, and let $f: I \rightarrow \mathbb{R}$ be absolutely monotonic (i.e., infinitely differentiable with all derivatives nonnegative). Then the Hermite interpolation of $f$ at $t_{1}, \ldots, t_{M}$ is a nonnegative linear combination of the polynomials

$$
t \mapsto \prod_{i=1}^{m}\left(t-t_{i}\right)
$$

for $0 \leq m<M$.

For a proof, see Lemma 10 in [16]. The precise interpretation of the lemma depends on the ordering of $t_{1}, \ldots, t_{M}$, but the lemma is true for every ordering. It follows from this lemma and (5.3) that $H_{k, M} \geq 0$ for $1 \leq k \leq M$. Furthermore, the same argument proves that $H_{0, M} \geq 0$ for $u_{0} \in(-\infty, 0]$, since $H_{0, M}$ is exactly the same sort of coefficient for interpolation at $u_{0}, \ldots, u_{M}$ rather than $u_{1}, \ldots, u_{M}$.

\section{INTERPOLATION BY ENTIRE FUNCTIONS OF EXPONENTIAL TYPE}

So far, we have a hypothetical definition of the auxiliary function $h$ in (5.1), but it is not clear that the limit in this equation even exists. To prove that it does exist and analyze its properties, we will extend the analysis from the previous section to carry out interpolation by entire functions of exponential type.

By the second identity in Lemma 5.1,

$$
e^{\alpha u_{0}}=\frac{1}{2 \pi i} \int_{\gamma} \sum_{k=0}^{\infty} \frac{e^{\alpha z}}{z-u_{k}} \prod_{j=k+1}^{\infty} \frac{1-u_{0} / u_{j}}{1-z / u_{j}} d z .
$$

We would like to interchange the sum and integral, but justifying this interchange requires careful bounds. The products

$$
\prod_{j=k+1}^{\infty}\left(1-u_{0} / u_{j}\right)
$$


are easily bounded: if we set $u_{0}=-|w|^{2}$, then we can rewrite these products as

$$
\prod_{j=k+1}^{\infty}\left(1-\left(\frac{\pi r|w|}{\lambda_{\lceil j / 2\rceil}}\right)^{2}\right)=c_{k} \frac{J_{n / 2}(\pi r|w|)^{2}}{(\pi r|w|)^{n} \prod_{j=1}^{k}\left(1-\frac{(\pi r|w|)^{2}}{\lambda_{\lceil j / 2\rceil}^{2}}\right)}
$$

by the Weierstrass product formula for the Bessel function (see $§ 15.41$ of [41]), where $c_{k}$ is a positive constant ensuring that the value at $w=0$ is 1 . Notice that the Bessel function is squared because we are interpolating to second order at each node, as reflected in (6.2) by the multiplicities in the ceiling function $\lceil j / 2\rceil$. (Second order interpolation is necessary to obtain the inequalities that $h$ must obey.) The functions on the right of (6.2) are positive definite on $\mathbb{R}^{n+2}$ by Proposition 3.1 and the fact that positive-definite functions are closed under multiplication. It follows that they are bounded in absolute value by their value when $w=0$, which is 1 .

We can also bound the effects of the $z-u_{k}$ factors in (6.1). Outside of the unit circle, the points in $\gamma$ are of the form $z=x \pm i x$ with $x<0$. At such points,

$$
\left|z-u_{k}\right|^{2}=\left(x-u_{k}\right)^{2}+x^{2} \geq u_{k}^{2} / 2
$$

with equality when $x=u_{k} / 2$, and hence

$$
\sum_{k=1}^{\infty} \frac{1}{\left|z-u_{k}\right|} \leq \sqrt{2} \sum_{k=1}^{\infty} \frac{1}{\left|u_{k}\right|}<\infty
$$

The products

$$
\prod_{j=k+1}^{\infty} \frac{1}{1-z / u_{j}}
$$

are the trickiest to handle, but we can bound them using the following lemma.

Lemma 6.1. Suppose $1 \leq M \leq \infty, 0 \leq k<M$, and $z=x \pm i x$ with $x<0$. Then

$$
\prod_{j=k+1}^{M} \frac{1}{\left|1-z / u_{j}\right|^{2}} \leq C^{\sqrt{|x|}}
$$

where $C$ is a constant that does not depend on $k, M$, or $x$.

Proof. We begin with

$$
\begin{aligned}
\left|1-z / u_{j}\right|^{2} & =\left(1-x / u_{j}\right)^{2}+\left(x / u_{j}\right)^{2} \\
& =1-2 x / u_{j}+2 x^{2} / u_{j}^{2} \\
& \geq 1 / 2
\end{aligned}
$$

with equality when $x=u_{j} / 2$, and the middle equation implies that $\left|1-z / u_{j}\right|^{2} \geq$ $1-2 x / u_{j}$ for all $x$. Thus,

$$
\prod_{j=k+1}^{M} \frac{1}{\left|1-z / u_{j}\right|^{2}} \leq 2^{O(\sqrt{|x|})} \prod_{\substack{j \text { such that } \\\left|u_{j}\right|>4|x|}} \frac{1}{1-2 x / u_{j}}
$$

where there are only $O(\sqrt{|x|})$ factors of 2 because $\left|u_{j}\right|$ grows quadratically as a function of $j$. Furthermore, the remaining indices $j$ satisfy

$$
\frac{1}{1-2 x / u_{j}} \leq 1+\frac{4 x}{u_{j}}
$$


because $(1-2 \varepsilon)(1+4 \varepsilon)>1$ for $0<\varepsilon<1 / 4$. It follows from this inequality and $1 /\left|u_{j}\right|=O\left(1 / j^{2}\right)$ that

$$
\prod_{j=k+1}^{M} \frac{1}{\left|1-z / u_{j}\right|^{2}} \leq 2^{O(\sqrt{|x|})} \prod_{j \geq 1}\left(1+\frac{K|x|}{j^{2}}\right)=2^{O(\sqrt{|x|})} \frac{\sinh (\pi \sqrt{K|x|})}{\pi \sqrt{K|x|}}
$$

for some positive constant $K$, which completes the proof because

$$
|\sinh v| \leq e^{|v|} / 2
$$

for all $v$.

Our estimates thus suffice to interchange the sum and integral in

$$
e^{\alpha u_{0}}=\frac{1}{2 \pi i} \int_{\gamma} \sum_{k=0}^{\infty} \frac{e^{\alpha z}}{z-u_{k}} \prod_{j=k+1}^{\infty} \frac{1-u_{0} / u_{j}}{1-z / u_{j}} d z
$$

by dominated convergence, because the exponential decay of $e^{\alpha z}$ outweighs the $C^{\sqrt{|x|}}$ growth from Lemma 6.1. Thus,

$$
e^{\alpha u_{0}}=\sum_{k=0}^{\infty} H_{k, \infty} \prod_{j=k+1}^{\infty}\left(1-\frac{u_{0}}{u_{j}}\right),
$$

where

$$
H_{k, \infty}=\frac{1}{2 \pi i} \int_{\gamma} \frac{e^{\alpha z} d z}{\left(z-u_{k}\right) \prod_{j=k+1}^{\infty}\left(1-z / u_{j}\right)} .
$$

Furthermore, Lemma 6.1 suffices to show that

$$
H_{k, \infty}=\lim _{M \rightarrow \infty} H_{k, M}
$$

by dominated convergence, and hence $H_{k, \infty} \geq 0$ for all $k$. The integral formula (6.3) for $H_{k, \infty}$ shows that it is a constant for $k \geq 1$ and a holomorphic function of $u_{0}$ for $k=0$.

As above, we set $u_{0}=-|w|^{2}$. Because

$$
p_{M}\left(u_{0}\right)=e^{\alpha u_{0}}-H_{0, M} \prod_{j=1}^{M}\left(1-\frac{u_{0}}{u_{j}}\right),
$$

the fact that $H_{0, M} \rightarrow H_{0, \infty}$ and the convergence of the infinite product imply that $\lim _{M \rightarrow \infty} p_{M}\left(u_{0}\right)$ exists. Thus, our definition of $h(w)$ as this limit in (5.1) yields

$$
h(w)=e^{\alpha u_{0}}-H_{0, \infty} \prod_{j=1}^{\infty}\left(1-\frac{u_{0}}{u_{j}}\right)=\sum_{k=1}^{\infty} H_{k, \infty} \prod_{j=k+1}^{\infty}\left(1-\frac{u_{0}}{u_{j}}\right) .
$$

We must still prove that

(1) the function $h$ is continuous, integrable, and positive definite,

(2) $h(x) \leq f(|x|)$ for all $x \in \mathbb{R}^{n}$,

(3) $h(x)=O\left((1+|x|)^{-(n+1)}\right)$,

(4) the support of $\widehat{h}$ is contained in $B_{r}^{n}(0)$, and

(5) the functions $f$ and $h$ agree at radius $\lambda_{m} /(\pi r)$ for each $m \geq 1$. 
We begin with (1). The function $h$ is not just continuous, but holomorphic. As we observed in (6.2), each of the products in (6.4) is a positive-definite function on $\mathbb{R}^{n+2}$ (and hence also when restricted to $\mathbb{R}^{n}$ ) by Proposition 3.1 , and thus the same is true of $h$ since the cone of positive-definite functions is closed under pointwise limits. Integrability follows from

$$
h(w)=e^{\alpha u_{0}}-H_{0, \infty} \prod_{j=1}^{\infty}\left(1-\frac{u_{0}}{u_{j}}\right)
$$

because the Gaussian and infinite product are integrable (for the latter see (6.5) below) while

$$
H_{0, \infty}=\frac{1}{2 \pi i} \int_{\gamma} \frac{e^{\alpha z} d z}{\left(z-u_{0}\right) \prod_{j=1}^{\infty}\left(1-z / u_{j}\right)} .
$$

is bounded as a function of $u_{0}$ on $(-\infty, 0]$ by Lemma 6.1 .

Furthermore, $h(w)=O\left((1+|w|)^{-(n+1)}\right)$ because

$$
\prod_{j=1}^{\infty}\left(1-\frac{u_{0}}{u_{j}}\right)=c_{0} \frac{J_{n / 2}(\pi r|w|)^{2}}{(\pi r|w|)^{n}}=O\left((1+|w|)^{-(n+1)}\right)
$$

by the usual asymptotics for Bessel functions (see $\S 7.21$ of [41]), while $e^{-\alpha|w|^{2}}$ decays even faster, and thus (3) holds.

The equality condition (5) holds because

$$
e^{-\alpha|w|^{2}}-h(w)=H_{0, \infty} \prod_{j=1}^{\infty}\left(1-\frac{u_{0}}{u_{j}}\right)
$$

This same equation yields the inequality (2) because $H_{0, \infty} \geq 0$ for all $u_{0} \in(-\infty, 0]$ while the product is nonnegative because each $u_{j}$ occurs with multiplicity two.

We must still check that the support of $\widehat{h}$ is contained in $B_{r}^{n}(0)$. First, note that setting $u_{0}=0$ in (6.4) shows that

$$
\sum_{k=1}^{\infty} H_{k, \infty}<\infty
$$

Each summand

$$
\prod_{j=k+1}^{\infty}\left(1-\frac{u_{0}}{u_{j}}\right)
$$

has Fourier transform with support in $B_{r}^{n}(0)$ (after setting $u_{0}=-|w|^{2}$ with $w \in \mathbb{R}^{n}$ ), by (6.2) and Corollary 4.2. We conclude that $\operatorname{supp}(\widehat{h}) \subseteq B_{r}^{n}(0)$ by the following lemma.

Lemma 6.2. Suppose $g_{1}, g_{2}, \ldots$ are integrable functions from $\mathbb{R}^{n}$ to $\mathbb{R}$ such that $\left|g_{k}\right| \leq 1$ everywhere and $\operatorname{supp}\left(\widehat{g_{k}}\right) \subseteq B_{r}^{n}(0)$ for all $k$. Let $g=\sum_{k \geq 1} c_{k} g_{k}$, where $c_{1}, c_{2}, \ldots$ satisfy $\sum_{k \geq 1}\left|c_{k}\right|<\infty$, and suppose $g$ is integrable. Then $\operatorname{supp}(\widehat{g}) \subseteq B_{r}^{n}(0)$ as well.

Proof. For each $\varepsilon>0$, let $i_{\varepsilon}$ be a smooth, radial function from $\mathbb{R}^{n}$ to $\mathbb{R}$ that is nonnegative, has integral 1, and is supported in $B_{\varepsilon}^{n}(0)$. Then its Fourier transform $\widehat{\imath}_{\varepsilon}$ is a Schwartz function, and the infinite sum

$$
g \widehat{\imath}_{\varepsilon}=\sum_{k \geq 1} c_{k} \widehat{\imath}_{\varepsilon} g_{k}
$$


converges in $L^{1}$. It follows that the sum over $k$ commutes with the Fourier transform, and hence

$$
\widehat{g} * i_{\varepsilon}=\sum_{k \geq 1} c_{k} \widehat{g}_{k} * i_{\varepsilon},
$$

where $*$ denotes convolution. The support of the right side is contained in $B_{r+\varepsilon}^{n}(0)$, while the left side converges pointwise to $\widehat{g}$ as $\varepsilon \rightarrow 0$ since $\widehat{g}$ is continuous and convolving with $i_{\varepsilon}$ is an approximate identity. Thus, $\operatorname{supp}(\widehat{g}) \subseteq B_{r}^{n}(0)$, as desired.

We have thus shown that the auxiliary function $h$ has all the properties (1) through (5), which completes the proof of Theorem 1.4.

The auxiliary function $h$ is presumably not optimal in general, but it is optimal subject to $\operatorname{supp}(\widehat{h}) \subseteq B_{r}^{n}(0)$ and $h(x)=O\left((1+|x|)^{-n-\delta}\right)$ for $x \in \mathbb{R}$ with $\delta>0$. This optimality follows immediately from Lemma 4.3:

Proposition 6.3. Let $f: \mathbb{R} \rightarrow \mathbb{R}$ be a Gaussian, let $\lambda_{1}<\lambda_{2}<\cdots$ be the positive roots of the Bessel function $J_{n / 2}$, choose $r$ so that $\operatorname{vol}\left(B_{r / 2}^{n}(0)\right)=\rho$, let $h$ be an even entire function of exponential type at most $2 \pi r$, and suppose that $h(x) \leq f(x)$ and $h(x)=O\left((1+|x|)^{-n-\delta}\right)$ for $x \in \mathbb{R}$ with $\delta>0$. Then

$$
\rho \widehat{h}(0)-h(0) \geq \frac{n}{2^{n-1}(n / 2) !^{2}} \sum_{m=1}^{\infty} \frac{\lambda_{m}^{n-2}}{J_{n / 2-1}\left(\lambda_{m}\right)^{2}} f\left(\frac{\lambda_{m}}{\pi r}\right) .
$$

Note that we do not require $h$ to be positive definite. This proposition is the analogue for energy minimization of the main theorem in [18] (see also [9]), and the analogue for Euclidean space of the optimality results in [3].

Proof. By Lemma 4.3,

$$
\rho \widehat{h}(0)-h(0)=\frac{n}{2^{n-1}(n / 2) !^{2}} \sum_{m=1}^{\infty} \frac{\lambda_{m}^{n-2}}{J_{n / 2-1}\left(\lambda_{m}\right)^{2}} h\left(\frac{\lambda_{m}}{\pi r}\right),
$$

and we simply bound $h$ from above by $f$.

\section{Asчmptotics}

To prove Theorems 1.2 and 1.3, all that remains is to carry out an asymptotic analysis to deduce them from Theorem 1.4. We begin with Theorem 1.2. Consider the Gaussian function $f(t)=e^{-\alpha t^{2}}$, and choose $r$ so that $\operatorname{vol}\left(B_{r / 2}^{n}(0)\right)=\rho$. We will assume that $(\alpha, \rho)$ is confined to a compact subset of $(0,4 \pi / e) \times(0, \infty)$, and given such a subset all our error terms will be uniform in $\alpha$ and $\rho$. We will estimate the sum

$$
\frac{n}{2^{n-1}(n / 2) !^{2}(\pi / \alpha)^{n / 2}} \sum_{m=1}^{\infty} \frac{\lambda_{m}^{n-2}}{J_{n / 2-1}\left(\lambda_{m}\right)^{2}} f\left(\frac{\lambda_{m}}{\pi r}\right),
$$

which is the bound from Theorem 1.4 with an extra factor of $(\pi / \alpha)^{n / 2}$ in the denominator, and we will show that it equals $\rho+o(1)$ as $n \rightarrow \infty$.

The key is to use the uniform asymptotic formulas for Bessel function zeros from $\S 10.21$ (viii) of [25]. The resulting expressions are somewhat cumbersome, and we will not write them all explicitly, but we will specify how to compute everything with reference to [25]. In particular, all references involving three numbers, such as 10.21.41, will be to equations in [25]. 
We will find that the largest terms in the sum (7.1) occur for $m$ near $c n$, where the constant $c$ depends on $\alpha$. For $\alpha<4 \pi / e$ the constant $c$ is positive, but for $\alpha \geq 4 \pi / e$ (the case of Theorem 1.3) it becomes 0 and the results change substantially.

The first step in analyzing (7.1) is to understand how large $\lambda_{m}$ is. By 10.21.41,

$$
\lambda_{m}=(n / 2) z_{m}+O(1 / n),
$$

where the constant in the big- $O$ is independent of $m$, the number $z_{m} \geq 1$ satisfies

$$
\frac{2}{3}\left(-\zeta_{m}\right)^{3 / 2}=\sqrt{z_{m}^{2}-1}-\operatorname{arcsec}\left(z_{m}\right)
$$

by 10.20 .3 , the number $\zeta_{m}<0$ satisfies

$$
\zeta_{m}=(n / 2)^{-2 / 3} a_{m}
$$

by 10.21 .41 , and $a_{m}$ is the $m$-th root of the Airy function Ai, with $0>a_{1}>a_{2}>\cdots$. Note that the $O(1 / n)$ error term in (7.2) is small enough that we can estimate $\lambda_{m}^{n}$ to within a $1+o(1)$ factor.

Equations (7.2) through (7.4) reduce the problem of estimating $\lambda_{m}$ to that of estimating $a_{m}$, and 9.9.6 tells us that

$$
a_{m}=-T\left(\frac{3 \pi(4 m-1)}{8}\right),
$$

where

$$
T(t)=t^{2 / 3}\left(1+\frac{5}{48} t^{-2}-\frac{5}{36} t^{-4}+O\left(t^{-6}\right)\right) .
$$

Using these formulas and (7.4), we find that if $m=c n+d \sqrt{n}$ with $c$ and $d$ bounded and $c$ bounded away from zero, then

$$
\frac{2}{3}\left(-\zeta_{m}\right)^{3 / 2}=2 \pi c\left(1+\frac{d}{c} n^{-1 / 2}-\frac{1}{4 c} n^{-1}+O\left(n^{-3 / 2}\right)\right),
$$

where the constant in the big- $O$ depends only on the bounds on $c$ and $d$. We will choose $c$ so that the largest terms in the sum (7.1) come from $m$ of this form.

We can identify the largest terms in the sum by analyzing the growth rate of

$$
\lambda_{m}^{n} f\left(\frac{\lambda_{m}}{\pi r}\right)
$$

because the remaining factors of $1 /\left(\lambda_{m}^{2} J_{n / 2-1}\left(\lambda_{m}\right)^{2}\right)$ will turn out to have lowerorder effects. If we use (7.2) to write (7.6) in terms of $z_{m}$ and use the estimate $r \sim \sqrt{2 n /(\pi e)}$ from Stirling's formula, ${ }^{2}$ then we find that

$$
\lambda_{m}^{n} f\left(\frac{\lambda_{m}}{\pi r}\right)=(n / 2)^{n}\left(z_{m} e^{-z_{m}^{2} \alpha e /(8 \pi)}+o(1)\right)^{n} .
$$

Thus, the exponential decay rate of the summand in (7.1) is determined by $z_{m} e^{-z_{m}^{2} \alpha e /(8 \pi)}$, which is maximized when $z_{m}=t_{m}$ with

$$
t_{m}=\sqrt{\frac{4 \pi}{\alpha e}} .
$$

This tells us that the greatest contribution to the sum should come from $m$ with $z_{m}=(1+o(1)) t_{m}$ as $n \rightarrow \infty$. (Note that $t_{m}>1$ because $\alpha<4 \pi / e$, in accordance

\footnotetext{
${ }^{2}$ Note that the estimate $\sqrt{2 n /(\pi e)}$ for $r$ is not precise enough to obtain the terms in (7.1) to within a better factor than $(1+o(1))^{n}$. Here we use it just to identify the largest terms in the sum, and we will use a higher-order Stirling approximation to $r$ when computing the final answer.
} 
with the restriction that $z_{m} \geq 1$. When $\alpha \geq 4 \pi / e$ the largest terms instead come from setting $z_{m}=1$.)

By (7.3) and (7.5), the value of $c$ that yields $z_{m}=t_{m}$ in the limit as $n \rightarrow \infty$ is

$$
c=\frac{\sqrt{\frac{4 \pi}{\alpha e}-1}-\operatorname{arcsec}\left(\sqrt{\frac{4 \pi}{\alpha e}}\right)}{2 \pi},
$$

and we fix that value of $c$ from this point on (while allowing $d$ to vary). Note that because $\alpha$ is bounded away from 0 and $4 \pi / e, c$ is bounded away from $\infty$ and 0 , as desired. Furthermore, one can use the equations listed above to compute explicit constants $k_{1}$ and $k_{2}$ in terms of $\alpha$ and $d$ such that

$$
z_{m}=t_{m}\left(1+k_{1} n^{-1 / 2}+k_{2} n^{-1}+O\left(n^{-3 / 2}\right)\right)
$$

when $m=c n+d \sqrt{n}$. We omit the complicated formulas here.

Using our equations so far, we can obtain uniform asymptotics to within a $1+o(1)$ factor for the terms

$$
\lambda_{m}^{n-2} f\left(\frac{\lambda_{m}}{\pi r}\right)
$$

when $m=c n+d \sqrt{n}$ with $c$ satisfying (7.7) and $d$ bounded. What remains is to deal with the factor of $J_{n / 2-1}\left(\lambda_{m}\right)^{2}$ in the denominator of the summand in (7.1).

It follows from 10.6.2 that

$$
J_{n / 2-1}\left(\lambda_{m}\right)=J_{n / 2}^{\prime}\left(\lambda_{m}\right)
$$

and 10.21.42 tells us that

$$
J_{n / 2}^{\prime}\left(\lambda_{m}\right)=-\frac{2}{(n / 2)^{2 / 3}} \frac{\operatorname{Ai}^{\prime}\left(a_{m}\right)}{z_{m} h_{m}}\left(1+O\left(n^{-2}\right)\right)
$$

with error term uniform in $m$, where by 10.21 .45

$$
h_{m}=\left(\frac{4 \zeta_{m}}{1-z_{m}^{2}}\right)^{1 / 4}=(1+o(1)) \pi^{1 / 6} 3^{1 / 6} 2^{1 / 2} c^{1 / 6} \alpha^{1 / 4}(4 \pi / e-\alpha)^{-1 / 4}
$$

as $m \rightarrow \infty$. To complete the analysis of (7.8), we need an estimate for $\operatorname{Ai}^{\prime}\left(a_{m}\right)$, which follows from 9.9.7:

$$
\operatorname{Ai}^{\prime}\left(a_{m}\right)=(-1)^{m-1} V\left(\frac{3 \pi(4 m-1)}{8}\right)
$$

where

$$
V(t)=\frac{t^{1 / 6}}{\sqrt{\pi}}\left(1+\frac{5}{48} t^{2}-\frac{1525}{4608} t^{4}+O\left(t^{-6}\right)\right) .
$$

Since $m=c n+d \sqrt{n}=(c+o(1)) n$, we find that

$$
\operatorname{Ai}^{\prime}\left(a_{m}\right)^{2}=(1+o(1))\left(\frac{3 c n}{2 \pi^{2}}\right)^{1 / 3} .
$$

To complete the proof of Theorem 1.2, we simply combine all the asymptotic formulas we have derived so far to estimate the terms in (7.1) when $m=c n+d \sqrt{n}$ with $d$ bounded. We find that

$$
\frac{n}{2^{n-1}(n / 2) !^{2}(\pi / \alpha)^{n / 2}} \cdot \frac{\lambda_{m}^{n-2}}{J_{n / 2-1}\left(\lambda_{m}\right)^{2}} f\left(\frac{\lambda_{m}}{\pi r}\right)=(\rho+o(1)) e^{-\pi K d^{2}} \sqrt{\frac{K}{n}},
$$


where

$$
K=\frac{4 \pi \alpha}{4 \pi / e-\alpha}
$$

In other words, the terms behave like a Gaussian in the variable $d$. If we convert the sum over $m$ with $|d| \leq R$ into an integral by viewing it as a Riemann sum, then it becomes

$$
(\rho+o(1)) \sqrt{K} \int_{-R}^{R} e^{-\pi K x^{2}} d x
$$

in the limit as $n \rightarrow \infty$ with $R$ fixed. (Note that the factor of $\sqrt{n}$ disappears due to the spacing in the Riemann sum.) Straightforward error bounds show that the $o(1)$ term in (7.9) is uniform in $\alpha$ and $\rho$ given our assumptions, but we do not obtain uniformity in $R$.

All that remains is to deal with the other terms in the sum, i.e., those for which $d$ is not bounded. Because these terms are nonnegative, we can obtain a lower bound by simply omitting them. Thus, (7.9) is a lower bound for (7.1) for each fixed $R$, and letting $R \rightarrow \infty$ shows that (7.1) is at least $\rho+o(1)$, because

$$
\sqrt{K} \int_{-\infty}^{\infty} e^{-\pi K x^{2}} d x=1
$$

More precisely,

$$
\sqrt{K} \int_{-R}^{R} e^{-\pi K x^{2}} d x=\int_{-R \sqrt{K}}^{R \sqrt{K}} e^{-\pi y^{2}} d y
$$

which converges uniformly to 1 as $R \rightarrow \infty$ because $K$ is bounded away from 0 . Combining this lower bound of $\rho+o(1)$ for $(7.1)$ with the expectation upper bound completes the proof of Theorem 1.2.

To prove Theorem 1.3, we simply bound the sum in Theorem 1.4 from below by the $m=1$ term using the same asymptotic formulas as above. Of course taking into account more terms would yield a more refined estimate, but even a single term suffices to obtain the correct exponential decay rate for the sum, and we see no need to analyze this bound more carefully given that it is almost certainly not sharp.

\section{The CONDITIONAL EXPECTATION BOUND}

In this section we prove an upper bound for energy that we call the conditional expectation bound. It refines the expectation bound by conditioning on having no short vectors in the lattice, and it shows that the expectation bound is not sharp for steep Gaussians, as described in Section 1.

Lemma 8.1. Let $n>1$ and $\rho>0$, let $f:(0, \infty) \rightarrow \mathbb{R}$ be such that $x \mapsto f(|x|)$ is integrable on $\mathbb{R}^{n} \backslash\{0\}$, and let $r \geq 0$ be such that $0 \leq \operatorname{vol}\left(B_{r}^{n}(0)\right)<2$. Then there exists a lattice in $\mathbb{R}^{n}$ of density $\rho$ and $f$-energy at most

$$
\frac{\rho}{1-\operatorname{vol}\left(B_{r}^{n}(0)\right) / 2} \int_{\mathbb{R}^{n} \backslash B_{r}^{n}(0)} f(|x|) d x .
$$

Proof. Let $\Lambda$ be a random lattice in $\mathbb{R}^{n}$ of density $\rho$, chosen according to the canonical probability measure on such lattices, and let $\mathbb{E}$ denote expectation with respect to that measure. Then for all integrable potential functions $g$, the $g$-energy $E_{g}(\Lambda)$ satisfies

$$
\mathbb{E}\left(E_{g}(\Lambda)\right)=\rho \int_{\mathbb{R}^{n}} g(|x|) d x
$$


by the Siegel mean value theorem.

Let $\chi_{r}$ be the characteristic function of $B_{r}^{n}(0)$. We will split the expected value

$$
\mathbb{E}\left(E_{f\left(1-\chi_{r}\right)}(\Lambda)\right)=\rho \int_{\mathbb{R}^{n} \backslash B_{r}^{n}(0)} f(|x|) d x
$$

into two pieces, depending on whether $\Lambda \cap B_{r}^{n}(0)=\{0\}$. Let $X$ denote the event that $\Lambda \cap B_{r}^{n}(0)=\{0\}$, let $\neg X$ denote the complementary event that $\Lambda \cap B_{r}^{n}(0) \neq\{0\}$, and let $p$ be the probability of $X$. Then the conditional expectations satisfy

$$
\mathbb{E}\left(E_{f\left(1-\chi_{r}\right)}(\Lambda)\right)=p \mathbb{E}\left(E_{f\left(1-\chi_{r}\right)}(\Lambda) \mid X\right)+(1-p) \mathbb{E}\left(E_{f\left(1-\chi_{r}\right)}(\Lambda) \mid \neg X\right) .
$$

Conditioned on $X$, the energies with respect to $f$ and $f\left(1-\chi_{r}\right)$ are identical, while the expectation conditioned on $\neg X$ is nonnegative, and thus

$$
\mathbb{E}\left(E_{f\left(1-\chi_{r}\right)}(\Lambda)\right) \geq p \mathbb{E}\left(E_{f}(\Lambda) \mid X\right) .
$$

As long as $p>0$, it follows that there exists a lattice $\Lambda$ with $f$-energy at most

$$
\frac{\rho}{p} \int_{\mathbb{R}^{n} \backslash B_{r}^{n}(0)} f(|x|) d x
$$

because that is an upper bound for the expected energy conditioned on $X$.

All that remains is to compute a lower bound for $p$. The number of nonzero lattice points in $B_{r}^{n}(0)$ is always an even integer, and by the Siegel mean value theorem its expectation is $\operatorname{vol}\left(B_{r}^{n}(0)\right)$. When the event $\neg X$ occurs, there are at least two nonzero lattice points in $B_{r}^{n}(0)$, and hence $\operatorname{vol}\left(B_{r}^{n}(0)\right) \geq 2(1-p)$. It follows that

$$
p \geq 1-\frac{\operatorname{vol}\left(B_{r}^{n}(0)\right)}{2}>0,
$$

and using this inequality in (8.1) completes the proof.

Computing the bound from Lemma 8.1 explicitly for a Gaussian in terms of the incomplete gamma function

$$
\Gamma(s, x)=\int_{x}^{\infty} e^{-t} t^{s} \frac{d t}{t}
$$

yields the following proposition, in which we take $\operatorname{vol}\left(B_{r}^{n}(0)\right)=1 / n$ for simplicity (although this choice does not exactly optimize the bound). Note that $0 \leq \Gamma(s, x) \leq$ $\Gamma(s, 0)=\Gamma(s)$ for $s>0$ and $x \geq 0$.

Proposition 8.2. Define $f: \mathbb{R} \rightarrow \mathbb{R}$ by $f(t)=e^{-\alpha t^{2}}$ with $\alpha>0$. Then for each $\rho>0$ and $n>1$, there exists a lattice in $\mathbb{R}^{n}$ of density $\rho$ and $f$-energy at most

$$
\frac{\rho}{1-1 /(2 n)}\left(\frac{\pi}{\alpha}\right)^{n / 2} \frac{\Gamma\left(n / 2, \alpha r^{2}\right)}{\Gamma(n / 2)},
$$

where $r>0$ satisfies $\operatorname{vol}\left(B_{r}^{n}(0)\right)=1 / n$. As $n \rightarrow \infty$ with $\alpha$ and $\rho$ fixed and $\alpha>\pi e$,

$$
\frac{\Gamma\left(n / 2, \alpha r^{2}\right)}{\Gamma(n / 2)}=\left(e^{1 / 2-\alpha /(2 \pi e)}+o(1)\right)^{n} .
$$

Proof. The bound from Lemma 8.1 is

$$
\frac{\rho}{1-\operatorname{vol}\left(B_{r}^{n}(0)\right) / 2} \int_{\mathbb{R}^{n} \backslash B_{r}^{n}(0)} f(|x|) d x=\frac{\rho}{1-1 /(2 n)} \int_{r}^{\infty} e^{-\alpha u^{2}} n \frac{\pi^{n / 2}}{(n / 2) !} u^{n-1} d u,
$$


and a change of variable from $u$ to $t=\alpha u^{2}$ yields the bound

$$
\frac{\rho}{1-1 /(2 n)}\left(\frac{\pi}{\alpha}\right)^{n / 2} \frac{\Gamma\left(n / 2, \alpha r^{2}\right)}{\Gamma(n / 2)}
$$

as stated above.

To compute the asymptotics, we can use the Laplace method to estimate the incomplete gamma function (see Chapter 4 of [4]). Specifically, this method shows that as $k \rightarrow \infty$,

$$
\frac{\int_{\beta+o(1)}^{\infty} e^{-k v} v^{k} d v}{\int_{0}^{\infty} e^{-k v} v^{k} d v}= \begin{cases}1+o(1) & \text { if } \beta<1, \text { and } \\ e^{k(1-\beta+o(1))} & \text { if } \beta>1\end{cases}
$$

The reason is that the primary contribution to the integral in the denominator comes from near $v=1$ and occurs on a scale of $1 / \sqrt{k}$. When $\beta<1$, the same contribution occurs in the numerator, while the primary contribution to the numerator comes from the left endpoint when $\beta>1$.

To apply these asymptotics, we write

$$
\frac{\Gamma\left(n / 2, \alpha r^{2}\right)}{\Gamma(n / 2)}=\frac{\int_{\alpha r^{2}}^{\infty} e^{-t} t^{n / 2-1} d t}{\int_{0}^{\infty} e^{-t} t^{n / 2-1} d t}=\frac{\int_{\alpha r^{2} /(n / 2-1)}^{\infty} e^{-(n / 2-1) v} v^{n / 2-1} d v}{\int_{0}^{\infty} e^{-(n / 2-1) v} v^{n / 2-1} d v}
$$

The equation $\operatorname{vol}\left(B_{r}^{n}(0)\right)=1 / n$ yields $r^{2} \sim n /(2 \pi e)$ as $n \rightarrow \infty$, and hence $\alpha r^{2} /(n / 2-1) \rightarrow \alpha /(\pi e)$. Thus, for $\alpha<\pi e$,

$$
\frac{\Gamma\left(n / 2, \alpha r^{2}\right)}{\Gamma(n / 2)}=1+o(1)
$$

as $n \rightarrow \infty$, while for $\alpha>\pi e$,

$$
\frac{\Gamma\left(n / 2, \alpha r^{2}\right)}{\Gamma(n / 2)}=\left(e^{1 / 2-\alpha /(2 \pi e)}+o(1)\right)^{n}
$$

as desired.

The factor of $1-\operatorname{vol}\left(B_{r}^{n}(0)\right) / 2$ in Lemma 8.1 plays an essential role in our proof, but we suspect that it may not be needed for the lemma to hold. One reason is that by using the methods of $[38,39]$ to average over lattices invariant under multiplication by the $k$-th roots of unity, we can replace this factor with $1-\operatorname{vol}\left(B_{r}^{n}(0)\right) / k$ whenever $n$ is a multiple of $\varphi(k)$ with $n>\varphi(k)$ and $0 \leq \operatorname{vol}\left(B_{r}^{n}(0)\right)<k$; here $\varphi$ is the Euler totient function, defined by $\varphi(k)=\#\{i \in\{1,2, \ldots, k\}: \operatorname{gcd}(i, k)=1\}$. Thus, for certain dimensions we can achieve a factor much closer to 1 , and we imagine that the same might hold for every dimension.

Another motivation is the following lemma:

Lemma 8.3. Let $f:(0, \infty) \rightarrow \mathbb{R}$ be such that $x \mapsto f(|x|)$ is integrable on $\mathbb{R}^{n} \backslash\{0\}$, let $h: \mathbb{R}^{n} \rightarrow \mathbb{R}$ be continuous, positive definite, and integrable and satisfy $h(x) \leq f(|x|)$ for all $x \in \mathbb{R}^{n} \backslash\{0\}$, and let $\rho>0$ and $r>0$ be such that $\operatorname{vol}\left(B_{r}^{n}(0)\right)=1 / \rho$. Then

$$
\rho \widehat{h}(0)-h(0) \leq \rho \int_{\mathbb{R}^{n} \backslash B_{r}^{n}(0)} f(|x|) d x .
$$

Recall that $\rho \widehat{h}(0)-h(0)$ is the lower bound for energy in Proposition 2.2. Thus, Lemma 8.3 says that the linear programming bound behaves as if there were a point 
configuration of energy

$$
\rho \int_{\mathbb{R}^{n} \backslash B_{r}^{n}(0)} f(|x|) d x
$$

when $\operatorname{vol}\left(B_{r}^{n}(0)\right)=1 / \rho$, regardless of whether such a configuration in fact exists. For comparison, when $\rho>1 / 2$ Lemma 8.1 guarantees the existence of a configuration of energy

$$
\frac{\rho}{1-1 /(2 \rho)} \int_{\mathbb{R}^{n} \backslash B_{r}^{n}(0)} f(|x|) d x
$$

which is slightly worse, and when $\rho \leq 1 / 2$ we cannot even apply Lemma 8.1 with $\operatorname{vol}\left(B_{r}^{n}(0)\right)=1 / \rho$.

Proof. As above, let $\chi_{r}$ be the characteristic function of $B_{r}^{n}(0)$. Then

$$
\begin{aligned}
\rho \int_{\mathbb{R}^{n} \backslash B_{r}^{n}(0)} f(|x|) d x & =\rho \int_{\mathbb{R}^{n}}\left(1-\chi_{r}\right) f \\
& \geq \rho \int_{\mathbb{R}^{n}}\left(1-\chi_{r}\right) h \\
& =\rho \widehat{h}(0)-\rho \int_{\mathbb{R}^{n}} \chi_{r} h \\
& =\rho \widehat{h}(0)-\rho \int_{\mathbb{R}^{n}} \widehat{\chi}_{r} \widehat{h},
\end{aligned}
$$

where the last equality follows from the Plancherel theorem. Note that $h$ is in $L^{2}\left(\mathbb{R}^{n}\right)$ because it is integrable and bounded.

Because $\widehat{\chi}_{r}$ is positive definite, it is never larger than $\widehat{\chi}_{r}(0)=\operatorname{vol}\left(B_{r}^{n}(0)\right)=1 / \rho$. Furthermore, $\widehat{h} \geq 0$ and $\widehat{h}$ is integrable (see, for example, Lemma 5.6.2 in [27]). Thus,

$$
\begin{aligned}
\rho \widehat{h}(0)-\rho \int_{\mathbb{R}^{n}} \widehat{\chi}_{r} \widehat{h} & \geq \rho \widehat{h}(0)-\int_{\mathbb{R}^{n}} \widehat{h} \\
& =\rho \widehat{h}(0)-h(0),
\end{aligned}
$$

as desired.

In terms of tempered distributions, Lemma 8.3 amounts to using the distribution $\delta_{0}+\rho-\rho \chi_{r}$ as a feasible point in the dual to the linear programming bound, but we have expressed it directly in terms of elementary manipulations so that we can apply it to auxiliary functions that are not necessarily Schwartz functions. Our choice of distribution is analogous to those used in [36] and [29], but it is slightly simpler in that those papers also include a spherical delta function at radius $r$. One could likely prove a better bound by taking this extra term into account, but we have opted for simplicity.

\section{Sphere packing and the Gaussian core model}

In this section, we describe a more precise connection between sphere packing and the Gaussian core model. It will be convenient to normalize the sphere packing problem in $\mathbb{R}^{n}$ as follows: what is the smallest $r>0$ for which there is a discrete subset $\mathcal{C}$ of $\mathbb{R}^{n}$ with density 1 and minimal distance at least $r \sqrt{n}$ (i.e., no two distinct 
points in $\mathcal{C}$ are separated by a distance of less than $r \sqrt{n})$ ? Centering spheres of radius $r \sqrt{n} / 2$ at the points of such a configuration yields a packing density of

$$
\operatorname{vol}\left(B_{r \sqrt{n} / 2}^{n}(0)\right)=\frac{\pi^{n / 2}}{(n / 2) !}\left(\frac{r \sqrt{n}}{2}\right)^{n}=\left(\frac{r \sqrt{2 \pi e}}{2}+o(1)\right)^{n}
$$

as $n \rightarrow \infty$. The best sphere packings known in high dimensions achieve packing density $(1 / 2+o(1))^{n}$, i.e., $r=1 / \sqrt{2 \pi e}+o(1)$, and determining whether this value of $r$ is optimal is a major open problem.

The following proposition gives an upper bound for the Gaussian energy of a sphere packing when the Gaussian is steep enough. The primary difficulty is ruling out large numbers of pairs of points just slightly further apart than the minimal distance, and a crude bound based on volume suffices.

Proposition 9.1. Fix $\alpha>0$ and $r>0$, and let $f(t)=e^{-\alpha t^{2}}$. If $2 \alpha r^{2} \geq 1$, then every discrete subset of $\mathbb{R}^{n}$ with density 1 and minimal distance at least $r \sqrt{n}$ has $f$-energy at most

$$
\left(3 e^{-\alpha r^{2}}+o(1)\right)^{n}
$$

as $n \rightarrow \infty$.

Proof. Let $\mathcal{C}$ be such a configuration. To estimate the $f$-energy of $\mathcal{C}$, we will show that

$$
\sum_{y \in \mathcal{C} \backslash\{x\}} e^{-\alpha|x-y|^{2}} \leq\left(3 e^{-\alpha r^{2}}+o(1)\right)^{n}
$$

uniformly for each $x \in \mathcal{C}$.

To bound this sum, we let $\varepsilon>0$ and look at concentric open balls $U_{0}, U_{1}, \ldots$ about $x$, where

$$
U_{i}=\left\{y \in \mathbb{R}^{n}:|y-x|<(1+\varepsilon)^{i} r \sqrt{n}\right\} .
$$

By hypothesis, there are no points of $\mathcal{C}$ in $U_{0}$ except $x$. Thus,

$$
\begin{aligned}
\sum_{y \in \mathcal{C} \backslash\{x\}} e^{-\alpha|x-y|^{2}} & =\sum_{k=0}^{\infty} \sum_{y \in \mathcal{C} \cap\left(U_{k+1} \backslash U_{k}\right)} e^{-\alpha|x-y|^{2}} \\
& \leq \sum_{k=0}^{\infty}\left|\mathcal{C} \cap\left(U_{k+1} \backslash U_{k}\right)\right| e^{-\alpha r^{2}(1+\varepsilon)^{2 k} n} \\
& \leq \sum_{k=0}^{\infty}\left|\mathcal{C} \cap U_{k+1}\right| e^{-\alpha r^{2}(1+\varepsilon)^{2 k} n}
\end{aligned}
$$

To bound $\left|\mathcal{C} \cap U_{k+1}\right|$, note that if we place a sphere of radius $r \sqrt{n} / 2$ at each point of $\mathcal{C} \cap U_{k+1}$, then these spheres do not overlap and all lie within a sphere of radius $(1+\varepsilon)^{k+1} r \sqrt{n}+r \sqrt{n} / 2$. Thus,

$$
\left|\mathcal{C} \cap U_{k+1}\right| \leq \frac{\operatorname{vol}\left(B_{(1+\varepsilon)^{k+1} r \sqrt{n}+r \sqrt{n} / 2}^{n}\right)}{\operatorname{vol}\left(B_{r \sqrt{n} / 2}^{n}\right)}=\left(2(1+\varepsilon)^{k+1}+1\right)^{n},
$$

and our upper bound becomes

$$
\sum_{k=0}^{\infty}\left(2(1+\varepsilon)^{k+1}+1\right)^{n} e^{-\alpha r^{2}(1+\varepsilon)^{2 k} n} .
$$


Because $2(1+\varepsilon)^{k+1}+1 \leq 3(1+\varepsilon)^{k+1}$ and $-\alpha r^{2}(1+\varepsilon)^{2 k} n<-\alpha r^{2}(1+2 k \varepsilon) n$, we obtain a simpler upper bound of

$$
\begin{aligned}
\sum_{k=0}^{\infty}\left(3(1+\varepsilon)^{k+1}\right)^{n} e^{-\alpha r^{2}(1+2 k \varepsilon) n} & =3^{n} e^{-\alpha r^{2} n}(1+\varepsilon)^{n} \sum_{k=0}^{\infty}\left((1+\varepsilon)^{n} e^{-2 \alpha r^{2} n \varepsilon}\right)^{k} \\
& =\frac{3^{n} e^{-\alpha r^{2} n}(1+\varepsilon)^{n}}{1-(1+\varepsilon)^{n} e^{-2 \alpha r^{2} n \varepsilon}}
\end{aligned}
$$

where the geometric series converges because our hypothesis that $2 \alpha r^{2} \geq 1$ implies that $(1+\varepsilon) e^{-2 \alpha r^{2} \varepsilon}<1$.

Thus, we obtain an energy upper bound of a constant times $3^{n} e^{-\alpha r^{2} n}(1+\varepsilon)^{n}$ for each fixed $\varepsilon$ as $n \rightarrow \infty$, and letting $\varepsilon \rightarrow 0$ shows that the energy is at most $\left(3 e^{-\alpha r^{2}}+o(1)\right)^{n}$.

The factor of $e^{-\alpha r^{2} n}$ in this bound is the dominant factor when $\alpha$ is large, and it is essentially optimal: given an upper bound of $e^{-\alpha s^{2} n}$ for energy as $n \rightarrow \infty$ for some constant $s$, a $1-o(1)$ fraction of points must have no neighbors at distance less than $(s+o(1)) \sqrt{n}$ (or else the energy would be too large), and we can then obtain minimal distance $(s+o(1)) \sqrt{n}$ by removing a negligible fraction of the points. In other words, every low-energy configuration can be modified to form a dense packing, which is a partial converse to Proposition 9.1.

By contrast, the factor of $3^{n}$ comes from the volume estimate (9.1). One could improve this factor by using a more sophisticated packing density bound in place of (9.1), but that would not suffice to eliminate it entirely. For comparison, the energy must be at least $K e^{-\alpha r^{2} n}$, where $K$ is the average kissing number (i.e., the average number of neighbors at distance $r \sqrt{n}$ ), and this factor of $K$ might grow exponentially with $n$.

If we apply Proposition 9.1 to the best packings currently known, with $r=$ $1 / \sqrt{2 \pi e}+o(1)$, then we obtain an upper bound of $\left(3 e^{-\alpha /(2 \pi e)}+o(1)\right)^{n}$ when $\alpha \geq \pi e$, compared with $\left(e^{-\alpha /(2 \pi e)} \sqrt{\pi e / \alpha}+o(1)\right)^{n}$ from Proposition 8.2. The dominant behavior as $\alpha \rightarrow \infty$ is the same, with the difference being the lower-order factors of 3 or $\sqrt{\pi e / \alpha}$. In particular, the coefficient of $-1 /(2 \pi e)$ for $\alpha$ in Proposition 8.2 cannot be improved unless there exist exponentially denser sphere packings than those currently known.

Another consequence of Proposition 9.1 is that lower bounds for energy yield upper bounds for the sphere packing density. Suppose there exist packings with $\rho=1$ and $r=\beta / \sqrt{2 \pi e}+o(1)$ as $n \rightarrow \infty$, i.e., packing density $(\beta / 2+o(1))^{n}$. Then Proposition 9.1 gives an energy upper bound of $\left(3 e^{-\alpha \beta^{2} /(2 \pi e)}+o(1)\right)^{n}$ when $\alpha$ is large enough, while Theorem 1.3 gives a lower bound of

$$
\left(\frac{1}{2} e^{1-\alpha e /(8 \pi)}+o(1)\right)^{n}
$$

Comparing these bounds yields

$$
\frac{1}{2} e^{1-\alpha e /(8 \pi)} \leq 3 e^{-\alpha \beta^{2} /(2 \pi e)}
$$

and as $\alpha \rightarrow \infty$ we find that

$$
-\frac{e}{8 \pi} \leq-\frac{\beta^{2}}{2 \pi e}
$$


In other words, $\beta \leq e / 2$, which means no sphere packing can have packing density greater than $(e / 4+o(1))^{n}$. This argument recovers Levenshtein's bound [24], which is no surprise given that similar Bessel function constructions prove this bound directly $[9,12,18]$. Levenshtein's bound is not the best upper bound known for the sphere packing density in high dimensions. The best bound known can be obtained from the linear programming bound $[23,17]$, and it is natural to hope for a corresponding lower bound for energy, but we do not have one.

\section{Open PROBlems}

Many problems remain open, most notably determining the true asymptotics for minimal energy and for the linear programming bound. We expect that both the upper and the lower bounds can be improved when $\alpha$ is large, and we have no idea which may be closer to the truth.

One natural extension of our work would be to consider other potential functions, particularly inverse power laws. As discussed in Section 1, the conditional expectation bound is sharp to within a constant factor for inverse power laws $t \mapsto 1 / t^{n+s}$ with $s>0$ fixed as $n \rightarrow \infty$, and that constant factor would become $1+o(1)$ if Theorem 1.2 could be extended to all $\alpha<\pi e$. We conjecture that it can be extended:

Conjecture 10.1. When $f(t)=e^{-\alpha t^{2}}$ with $0<\alpha<\pi e$, the minimal f-energy in $\mathbb{R}^{n}$ for configurations of density $\rho$ is $(\rho+o(1))(\pi / \alpha)^{n / 2}$ as $n \rightarrow \infty$ with $\alpha$ and $\rho$ fixed, or more generally with $(\alpha, \rho)$ confined to a compact subset of $(0, \pi e) \times(0, \infty)$.

The conditional expectation bound shows that the bound $\pi e$ for $\alpha$ cannot be increased in Conjecture 10.1. What happens beyond that point? We would guess that there is some range of $\alpha$ over which the conditional expectation bound is asymptotically sharp. Perhaps it is sharp for all $\alpha>\pi e$, or perhaps there are further phase transitions when $\alpha$ is large, after which other bounds take over.

Note that the transition at $\pi e$ affects only the asymptotics for energy, and not the form of the ground states. Specifically, for fixed $\alpha>\pi e$ and $\rho$, a random lattice in $\mathbb{R}^{n}$ of density $\rho$ has energy at most

$$
\rho(\pi / \alpha)^{n / 2}\left(e^{1 / 2-\alpha /(2 \pi e)}+o(1)\right)^{n}
$$

with probability $1-o(1)$ as $n \rightarrow \infty$, because the bound in Proposition 8.2 is the expectation conditioned on an event of probability $1-o(1)$. (If there were a more than $o(1)$ chance of greater energy, then that alone would increase the expectation.) Thus, we have not disproved the hypothesis that random lattices asymptotically minimize Gaussian energy in high dimensions for all $\alpha$, as they do for $\alpha<4 \pi / e$. We suspect that this hypothesis is false, but we cannot propose any better constructions. As $\alpha$ grows, we expect that these problems will become increasingly difficult to resolve.

\section{REFERENCES}

[1] G. E. Andrews, R. Askey, and R. Roy, Special Functions, Encyclopedia of Mathematics and its Applications 71, Cambridge University Press, Cambridge, 1999. MR1688958 doi:10.1017/CBO9781107325937

[2] R. Ben Ghanem and C. Frappier, Explicit quadrature formulae for entire functions of exponential type, J. Approx. Theory 92 (1998), no. 2, 267-279. MR1604935 doi:10.1006/jath.1997.3122 
[3] P. G. Boyvalenkov, P. D. Dragnev, D. P. Hardin, E. B. Saff, and M. M. Stoyanova, Universal lower bounds for potential energy of spherical codes, Constr. Approx. 44 (2016), no. 3, 385-415. MR3562402 doi:10.1007/s00365-016-9327-5

[4] N. G. de Bruijn, Asymptotic methods in analysis, corrected reprint of the third edition, Dover Publications, Inc., New York, 1981. MR0671583

[5] E. Carneiro and F. Littmann, Extremal functions in de Branges and Euclidean spaces, II, Amer. J. Math. 139 (2017), no. 2, 525-566. MR3636639 doi:10.1353/ajm.2017.0014 arXiv:1508.02436

[6] E. Carneiro, F. Littmann, and J. D. Vaaler, Gaussian subordination for the Beurling-Selberg extremal problem, Trans. Amer. Math. Soc. 365 (2013), no. 7, 3493-3534. MR3042593 doi:10.1090/S0002-9947-2013-05716-9 arXiv:1008.4969

[7] E. Carneiro and J. D. Vaaler, Some extremal functions in Fourier analysis. II, Trans. Amer. Math. Soc. 362 (2010), no. 11, 5803-5843. MR2661497 doi:10.1090/S0002-9947-2010-04886-X arXiv:0809.4050

[8] E. Carneiro and J. D. Vaaler, Some extremal functions in Fourier analysis, III, Constr. Approx. 31 (2010), no. 2, 259-288. MR2581230 doi:10.1007/s00365-009-9050-6 arXiv:0809.4053

[9] H. Cohn, New upper bounds on sphere packings II, Geom. Topol. 6 (2002), 329-353. MR1914571 doi:10.2140/gt.2002.6.329 arXiv:math/0110010

[10] H. Cohn, Order and disorder in energy minimization, Proceedings of the International Congress of Mathematicians, Volume IV, 2416-2443, Hindustan Book Agency, New Delhi, 2010. MR2827978 doi:10.1142/9789814324359_0152 arXiv:1003.3053

[11] H. Cohn, Packing, coding, and ground states, in Mathematics and materials (M. J. Bowick, D. Kinderlehrer, G. Menon, and C. Radin, eds.), 45-102, IAS/Park City Math. Ser. 23, Amer. Math. Soc., Providence, RI, 2017. MR3700014 arXiv:1603.05202

[12] H. Cohn and N. Elkies, New upper bounds on sphere packings I, Ann. of Math. (2) 157 (2003), no. 2, 689-714. MR1973059 doi:10.4007/annals.2003.157.689 arXiv:math/0110009

[13] H. Cohn and A. Kumar, Universally optimal distribution of points on spheres, J. Amer. Math. Soc. 20 (2007), no. 1, 99-148. MR2257398 doi:10.1090/S0894-0347-06-00546-7 arXiv:math/0607446

[14] H. Cohn, A. Kumar, S. D. Miller, D. Radchenko, and M. Viazovska, The sphere packing problem in dimension 24, Ann. of Math. (2) 185 (2017), no. 3, 1017-1033. MR3664817 doi:10.4007/annals.2017.185.3.8 arXiv:1603.06518

[15] H. Cohn, A. Kumar, and A. Schürmann, Ground states and formal duality relations in the Gaussian core model, Phys. Rev. E (3) 80 (2009), no. 6, 061116, 7 pp. doi:10.1103/PhysRevE.80.061116 arXiv:0911.2169

[16] H. Cohn and J. Woo, Three-point bounds for energy minimization, J. Amer. Math. Soc. 25 (2012), no. 4, 929-958. MR2947943 doi:10.1090/S0894-0347-2012-00737-1 arXiv:1103.0485

[17] H. Cohn and Y. Zhao, Sphere packing bounds via spherical codes, Duke Math. J. 163 (2014), no. 10, 1965-2002. MR3229046 doi:10.1215/00127094-2738857 arXiv:1212.5966

[18] D. V. Gorbachev, An extremal problem for entire functions of exponential spherical type, which is connected with the Levenshtern bound for the density of a packing of $\mathbb{R}^{n}$ by balls (Russian), Izv. Tul. Gos. Univ. Ser. Mat. Mekh. Inform. 6 (2000), no. 1, Matematika, 71-78. MR2018751

[19] S. W. Graham and J. D. Vaaler, A class of extremal functions for the Fourier transform, Trans. Amer. Math. Soc. 265 (1981), no. 1, 283-302. MR0607121 doi:10.2307/1998495

[20] J. J. Holt and J. D. Vaaler, The Beurling-Selberg extremal functions for a ball in Euclidean space, Duke Math. J. 83 (1996), no. 1, 202-248. MR1388849 doi:10.1215/S0012-7094-96-08309$\mathrm{X}$

[21] L. Hörmander, The Analysis of Linear Partial Differential Operators I: Distribution Theory and Fourier Analysis, reprint of the second edition, Classics in Mathematics, Springer-Verlag, Berlin, 2003. MR1996773 doi:10.1007/978-3-642-61497-2

[22] R. A. Horn and C. R. Johnson, Matrix Analysis, second edition, Cambridge University Press, 2013. MR2978290

[23] G. A. Kabatyanskii and V. I. Levenshtein, Bounds for packings on a sphere and in space (Russian), Problemy Peredači Informacii 14 (1978), no. 1, 3-25; English translation in Problems of Information Transmission 14 (1978), no. 1, 1-17. MR0514023

[24] V. I. Levenštel̆n, On bounds for packings in n-dimensional Euclidean space (Russian), Dokl. Akad. Nauk SSSR 245 (1979), no. 6, 1299-1303; English translation in Soviet Math. Dokl. 20 (1979), no. 2, 417-421. MR529659 
[25] F. W. J. Olver, D. W. Lozier, R. F. Boisvert, and C. W. Clark, NIST Handbook of Mathematical Functions, National Institute of Standards and Technology, U.S. Department of Commerce, Washington, DC and Cambridge University Press, Cambridge, 2010. MR2723248 http: //dlmf.nist.gov/

[26] G. Poltyrev, On coding without restrictions for the AWGN channel, IEEE Trans. Inform. Theory 40 (1994), no. 2, 409-417. doi:10.1109/18.312163

[27] J. Ramanathan, Methods of Applied Fourier Analysis, Applied and Numerical Harmonic Analysis, Birkhäuser Boston, Inc., Boston, MA, 1998. MR1632358 doi:10.1007/978-1-46121756-5

[28] P. Sarnak and A. Strömbergsson, Minima of Epstein's zeta function and heights of flat tori, Invent. Math. 165 (2006), no. 1, 115-151. MR2221138 doi:10.1007/s00222-005-0488-2

[29] A. Scardicchio, F. H. Stillinger, and S. Torquato, Estimates of the optimal density of sphere packings in high dimensions, J. Math. Phys. 49 (2008), no. 4, 043301, 15 pp. MR2412293 doi:10.1063/1.2897027 arXiv:0705.1482

[30] I. J. Schoenberg, Positive definite functions on spheres, Duke Math. J. 9 (1942), 96-108. MR0005922 doi:10.1215/S0012-7094-42-00908-6

[31] A. Selberg, Collected Papers II, reprint of the 1991 edition, Springer Collected Works in Mathematics, Springer, Heidelberg, 2014. MR3308963

[32] C. L. Siegel, A mean value theorem in geometry of numbers, Ann. of Math. (2) 46 (1945), 340-347. MR0012093 doi:10.2307/1969027

[33] B. Simon, Real Analysis, A Comprehensive Course in Analysis, Part 1, American Mathematical Society, Providence, RI, 2015. MR3408971

[34] F. H. Stillinger, Phase transitions in the Gaussian core system, J. Chem. Phys. 65 (1976), no. 10, 3968-3974. doi:10.1063/1.432891

[35] G. Szegö, Orthogonal Polynomials, fourth edition, AMS Colloquium Publications 23, American Mathematical Society, Providence, RI, 1975. MR0372517

[36] S. Torquato and F. H. Stillinger, New conjectural lower bounds on the optimal density of sphere packings, Experiment. Math. 15 (2006), no. 3, 307-331. MR2264469 http://projecteuclid. org/euclid.em/1175789761 arXiv:math/0508381

[37] J. D. Vaaler, Some extremal functions in Fourier analysis, Bull. Amer. Math. Soc. (N.S.) 12 (1985), no. 2, 183-216. MR0776471 doi:10.1090/S0273-0979-1985-15349-2

[38] S. Vance, Improved sphere packing lower bounds from Hurwitz lattices, Adv. Math. 227 (2011), 2144-2156. MR2803798 doi:10.1016/j.aim.2011.04.016 arXiv:1105.3779

[39] A. Venkatesh, A note on sphere packings in high dimension, Int. Math. Res. Not. IMRN 2013 (2013), 1628-1642. MR3044452 doi:10.1093/imrn/rns096

[40] M. S. Viazovska, The sphere packing problem in dimension 8, Ann. of Math. (2) 185 (2017), no. 3, 991-1015. MR3664816 doi:10.4007/annals.2017.185.3.7 arXiv:1603.04246

[41] G. N. Watson, A Treatise on the Theory of Bessel Functions, reprint of the second edition, Cambridge Mathematical Library, Cambridge University Press, Cambridge, 1995. MR1349110

[42] D. V. Widder, The Laplace Transform, Princeton University Press, Princeton, New Jersey, 1941. MR0005923

Microsoft Research New England, One Memorial Drive, Cambridge, MA 02142

E-mail address: cohn@microsoft.com

Department of Mathematics, Princeton University, Princeton NJ 08544

E-mail address: mdc4@math.princeton.edu 